Article

\title{
Numerical Solutions for Multi-Term Fractional Order Differential Equations with Fractional Taylor Operational Matrix of Fractional Integration
}

\author{
İbrahim Avcı *(D) and Nazim I. Mahmudov \\ Department of Mathematics, Eastern Mediterranean University, Famagusta, TR 99628, Northern Cyprus, \\ via Mersin-10, Turkey; nazim.mahmudov@emu.edu.tr \\ * Correspondence: ibrahim.avci@emu.edu.tr
}

Received: 15 December 2019; Accepted: 31 December 2019; Published: 7 January 2020

\begin{abstract}
In this article, we propose a numerical method based on the fractional Taylor vector for solving multi-term fractional differential equations. The main idea of this method is to reduce the given problems to a set of algebraic equations by utilizing the fractional Taylor operational matrix of fractional integration. This system of equations can be solved efficiently. Some numerical examples are given to demonstrate the accuracy and applicability. The results show that the presented method is efficient and applicable.
\end{abstract}

Keywords: fractional differential equations; numerical solutions; Riemann-Liouville fractional integral; Caputo fractional derivative; fractional Taylor vector

MSC: 26A33, 34A08

\section{Introduction}

Fractional calculus is an emerging field of mathematics, which is a generalisation of differentiation and integration to non-integer orders. The history of fractional calculus is almost as long as the history of classical calculus, beginning with some speculations of Leibniz $(1695,1697)$ and Euler $(1730)$. However, fractional calculus and fractional differential equations (FDEs) are increasingly becoming popular in recent years. The progressively developing history of this old and yet novel topic can be found in [1-5]. In fact, fractional calculus provides the mathematical modeling of some important phenomena like social and natural in a more powerful way than the classical calculus. During the last few decades, many applications were reported in many branches of science and engineering such as chaotic systems [6,7], fluid mechanics [8], viscoelasticity [9], optimal control problems [10,11], chemical kinetics [12,13], electrochemistry [14], biology [15], physics [16], bioengineering [17], finance [18], social sciences [19], economics [20,21], optics [22], chemical reactions [23], rheology [24], and so on. Due to the importance of FDEs, the solutions of them are attracting widespread interest. On the other hand, analytical solutions are not always possible for solving them. Therefore, numerical techniques becomes more important for solving such equations.

There are various numerical methods have been developed for solving FDEs in literature such as predictor-corrector method [25], Laplace transforms [26], Taylor collocation method [27], variational iteration method and homotopy perturbation method [8] (Chapter 6), Adomian decomposition method [28], Tau method [29], inverse Laplace transform [30], Haar wavelet collocation method [31], generalized block pulse operational matrix [32], shifted Legendre-tau method [33], fractional multi-step differential transformed method [34], q-homotopy analysis transform method [35], conformable Laplace transform [36], fractional B-splines collocation method [37], finite difference method [38], homotopy analysis method [39] and so on. 
Multi-term fractional differential equations are one of the most important type of FDEs, which is a system of mixed fractional and ordinary differential equations and involving more than one fractional differential operators. Nowadays, they are widely appearing for modelling of many important processes, especially for multirate systems. Their numerical solution is then a strong subject that deserves high attention. In this paper, motivated by the results reported in $[40,41]$ for solving a smaller class of problems where the highest order of derivative is an integer and involving at most one noninteger order derivative, we go further and establish a method for numerical solutions for higher order and arbitrary multi-term fractional differential equations which have a general form

$$
D^{\alpha} y(t)=f\left(t, y(t), D^{\beta_{0}} y(t), D^{\beta_{1}} y(t), \ldots, D^{\beta_{k}} y(t)\right), t \in[0, R]
$$

where $D^{\alpha}$ representing the Caputo fractional derivative of order $\alpha>0$ and we assume that $0<\beta_{0}<$ $\beta_{1}<\ldots<\beta_{k}<\alpha, y^{(p)}=Y_{p}, p=0,1, \ldots n$ where $n-1<\alpha<n$.

Multi-term fractional order differential equations also have useful properties and they can describe complex multi-rate physical processes in a various way and can be applied in many fields, see e.g., [2,4,26,42]. Basset [43] and Bagley-Torvik [44] equations can be given as important examples for smaller class of multi-term fractional differential equations. Existence, uniqueness and stability of solution for multi-term fractional differential equations are discussed in [45-49]. Because of difficulty of finding the exact solutions for such equations, many new numerical techniques have been developed to investigate the numerical solutions such as Adams method [50], Haar wavelet method [51], differential transform method [52], Adams-Bashforth-Moulton method [53], collocation method based on shifted Chebyshev polynomials of the first kind [54], Boubaker polynomials method [55], matrix Mittag-Leffler functions [56], differential transform method [57] and so on.

Our main purpose is to present an effective, reliable method to approximate initial value problem for the Equation (1). In order to reach this aim, we rewrite and focus the general type of Caputo multi-term fractional differential equation given in Equation (1) in the following linear form

$$
D^{\alpha} y(t)=\sum_{i=0}^{k} u_{i} D^{\beta_{i}} y(t)+u_{k+1} y(t)+f(t), \quad 0 \leq t \leq R,
$$

subject to the

$$
\begin{aligned}
y^{(p)}(0) & =Y_{p}, p=0,1, \ldots, n-1 \text { where } n-1<\alpha<n \\
u_{i}(i & =0,1, \ldots, k) \text { are known coefficients and } \\
0 & <\beta_{0}<\beta_{1}<\ldots<\beta_{k}<\alpha
\end{aligned}
$$

Here, we also state that the highest order $\alpha$ need not to be an integer. This equation is important in applications due to the fact it can treat the problems with fractional force, therefore it is suitable for being treated within fractional operators of Caputo type.

In this work, a numerical approach based on fractional Taylor vector is proposed to solve the initial value problem of general type of multi-term fractional differential equations which is given in Equations (2) and (3). The core idea of this method is to employ the operational matrix of fractional integration based on fractional Taylor vector to given problem and reduce it to a set of algebraic equations which can be efficiently solved.

The structure of the manuscript is organized as follows. In Section 2, we briefly introduce some preliminary ideas of fractional calculus and necessary definitions. In Section 3, an operational matrix of fractional integration based on fractional taylor vector is derived. In Section 4, we present the numerical algorithm to solve the given equation and a pseudo-code for matlab is also provided in Algorithm 1. In Section 5, the presented method is applied to six examples to demonstrate the efficiency. A final conclusion is presented in the last section. 


\section{Preliminary Knowledge}

In this section, we recall some fundamental definitions and preliminary facts of fractional calculus.

\subsection{The Fractional Integral and Derivative}

Definition 1. The Riemann-Liouville fractional integral to order $\alpha$ of an integrable function $y(t)$ is defined to be

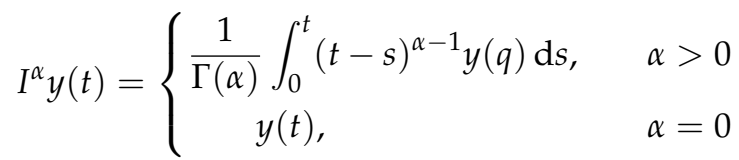

When applied to a power function, it yields the following result:

$$
I^{\alpha}(t)^{c}=\frac{\Gamma(c+1)}{\Gamma(c+\alpha+1)}(t)^{c+\alpha}, \alpha \geq 0, c>-1
$$

The operator has a semigroup property, namely

$$
I^{\alpha} I^{\beta} y(t)=I^{\beta} I^{\alpha} y(t), \alpha, \beta>0
$$

and it is linear, namely

$$
I^{\alpha}\left(A_{1} y_{1}(t)+A_{2} y_{2}(t)\right)=A_{1} I^{\alpha} y_{1}(t)+A_{2} I^{\alpha} y_{2}(t)
$$

for any two functions $y_{1}, y_{2}$ and constants $A_{1}, A_{2}$.

Definition 2. The fractional derivative of $y(t)$ of the order $\alpha$ in the Caputo sense is given as

$$
D^{\alpha} y(t)=I^{j-\alpha}\left(\frac{\mathrm{d}^{j}}{\mathrm{~d} t^{j}} y(t)\right), j-1<\alpha \leq j, j \in \mathbb{N}
$$

\subsection{Some Properties}

1. The Riemann-Liouville fractional integral and Caputo fractional derivative do not usually commute with each other. The following Newton-Leibniz identity gives an important relation between them:

$$
I^{\alpha}\left(D^{\alpha} y(t)\right)=y(t)-\sum_{i=0}^{j-1} y^{(i)}(0) \frac{t^{i}}{i !}
$$

2. The Caputo fractional derivative also has the following substitution identity. If we write $y_{1}(q)=$ $y(q R)$ and $q=t / R$, then

$$
D^{\alpha} y(t)=\frac{1}{R^{\alpha}} D^{\alpha} y_{1}(q)
$$

where $j-1<\alpha \leq j, j \in \mathbb{N}$

\section{Operational Matrix of Fractional Integration for Fractional Taylor Vector}

\subsection{Fractional Taylor Basis Vector}

We shall make use of the fractional Taylor vector,

$$
T_{m \delta}(t)=\left[1, t^{\delta}, t^{2 \delta}, \ldots, t^{m \delta}\right]
$$

for $m \in \mathbb{N}$ and $\delta>0$ in the work of this paper. 


\subsection{Approximation of Function}

Suppose that $T_{m \delta}(t) \subset H$, where $H$ is the space of all square integrable functions on the interval $[0,1]$. For any $y \in H$, since $S=\operatorname{span}\left\{1, t^{\delta}, t^{2 \delta}, \ldots, t^{m \delta}\right\}$ is a finite dimensional vector space in $H$, then, $y$ has a unique best approximation $y_{*} \in S$, so that

$$
\forall \widehat{y} \in S,\left\|y-y_{*}\right\| \leq\|y-\widehat{y}\|
$$

Therefore, the function $y$ is approximated by fractional Taylor vector as following

$$
y \simeq y_{*}=\sum_{i=0}^{m} c_{i} t^{i \delta}=C^{T} T_{m \delta}(t)
$$

where $T_{m \delta}(t)$ denote the fractional Taylor vector and

$$
C^{T}=\left[c_{0}, c_{1}, c_{2}, \ldots, c_{m}\right]
$$

are the unique coefficients.

\subsection{Fractional Taylor Operational Matrix of Integration}

By using the property of Riemann-Liouville fractional integral given in Equations (5) and (9), we get

$$
\begin{aligned}
I^{\alpha}\left(T_{m \delta}(t)\right) & =\left[\frac{1}{\Gamma(\alpha+1)} t^{\alpha}, \frac{\Gamma(\delta+1)}{\Gamma(\delta+\alpha+1)} t^{\delta+\alpha}, \frac{\Gamma(2 \delta+1)}{\Gamma(2 \delta+\alpha+1)} t^{2 \delta+\alpha}, \ldots, \frac{\Gamma(m \delta+1)}{\Gamma(m \delta+\alpha+1)} t^{m \delta+\alpha}\right] \\
& =t^{\alpha} M_{\alpha} T_{m \delta}(t)
\end{aligned}
$$

where

$$
M_{\alpha}=\operatorname{diag}\left[\frac{1}{\Gamma(\alpha+1)}, \frac{\Gamma(\delta+1)}{\Gamma(\delta+\alpha+1)}, \frac{\Gamma(2 \delta+1)}{\Gamma(2 \delta+\alpha+1)}, \ldots, \frac{\Gamma(m \delta+1)}{\Gamma(m \delta+\alpha+1)}\right]
$$

denotes the operational matrix of integration.

If we define $G_{\alpha}$ as

$$
G_{\alpha}=\left[\frac{1}{\Gamma(\alpha+1)}, \frac{\Gamma(\delta+1)}{\Gamma(\delta+\alpha+1)}, \frac{\Gamma(2 \delta+1)}{\Gamma(2 \delta+\alpha+1)}, \ldots, \frac{\Gamma(m \delta+1)}{\Gamma(m \delta+\alpha+1)}\right]
$$

then, we can rewrite the Equation (10) as

$$
I^{\alpha}\left(T_{m \delta}(t)\right)=t^{\alpha} G_{\alpha} * T_{m \delta}(t)
$$

where $*$ denotes the operation of multiplying matrices term by term.

\section{The Numerical Algorithm}

In this section, to solve the given multi-term fractional differential equation in Equations (2) and (3), we employ the fractional Taylor method. The algorithm of method is given below.

Firstly, by using the transformation $q=t / R$, we replace the variable $t \in[0, R]$ with $q \in[0,1]$. Now, by using Equation (8) in Equation (2), we get

$$
\frac{1}{R^{\alpha}} D^{\alpha} y_{1}(q)=\sum_{i=0}^{k} \frac{1}{R^{\beta_{i}}} u_{i} D^{\beta_{i}} y_{1}(q)+u_{k+1} y_{1}(q)+f_{1}(q), 0 \leq s \leq 1
$$


where $f_{1}(q)=f(q R)$ and $y_{1}(q)=y(q R)$. Similar to Equation (10) we approximate the $y_{1}(q)$ as

$$
y_{1}(q)=\sum_{i=0}^{m} c_{i} q^{i \delta}=C^{T} T_{m \delta}(q)
$$

such that $T_{m \delta}(q)=\left[1, q^{\delta}, q^{2 \delta}, \ldots, q^{m \delta}\right]^{T}$ is the fractional Taylor vector and the unique coefficients $C^{T}$ is given in Equation (11).

Next, applying the Riemann-Liouville fractional integral on both side of (14), we get

$$
\begin{aligned}
\frac{1}{R^{\alpha}}\left[y_{1}(q)-\sum_{j=0}^{n-1} y_{1}^{(j)}\left(0^{+}\right) \frac{t^{j}}{j !}\right] & =\sum_{i=0}^{k} \frac{1}{R^{\beta_{i}}} u_{i} I^{\alpha-\beta_{i}}\left[y_{1}(q)-\sum_{j=0}^{n_{i}-1} y_{1}^{(j)}\left(0^{+}\right) \frac{t^{j}}{j !}\right] \\
& +u_{k+1} I^{\alpha} y_{1}(q)+I^{\alpha} f_{1}(q)
\end{aligned}
$$

where $y^{(p)}(0)=V_{p}, p=0,1, \ldots, n-1$ where $n_{i}-1<\beta_{i}<n_{i}$.

Hence, by substituting initial conditions (3), we get

$$
\frac{1}{R^{\alpha}}\left[y_{1}(q)\right]=\sum_{i=0}^{k} \frac{1}{R^{\beta_{i}}} u_{i} I^{\alpha-\beta_{i}}\left[y_{1}(q)\right]+u_{k+1} I^{\alpha} y_{1}(q)+h_{1}(q)
$$

such that $h_{1}(q)=I^{\alpha} f_{1}(q)+\frac{1}{R^{\alpha}}\left(\sum_{j=0}^{n-1} V_{j} t_{j !}^{j}\right)+\sum_{i=0}^{k} \frac{1}{R^{\beta_{i}}} u_{i} I^{\alpha-\beta_{i}}\left(\sum_{j=0}^{n_{i}-1} V_{j} t^{j}\right)$.

Now, by using the Equation (12), we approximate the fractional order integrals in Equation (17) and we have

$$
\begin{aligned}
\frac{1}{R^{\alpha}}\left[C^{T} T_{m \delta}(q)\right] & =\sum_{i=0}^{k} \frac{1}{R^{\beta}} u_{i} C^{T} q^{\alpha-\beta_{i}}\left(G_{\alpha-\beta_{i}} * T_{m \delta}(q)\right) \\
& +u_{k+1} q^{\alpha} C^{T}\left(G_{\alpha} * T_{m \delta}(q)\right)+h_{1}(q)
\end{aligned}
$$

Finally, by taking the collocation points $q_{j}=j / m(j=0,1, \ldots, m)$ in Equation (18), we get $m+1$ linear algebraic equations. This linear system can be solved for the unknown vector $C^{T}$. Consequently, $y_{1}(q)$ can be approximated by Equation (15).

\section{MATLAB Implementation of Method}

The pseudocode given in Algorithm 1 below allows us to use proposed method in MATLAB for obtain a numerical solution of given problem [58]. 


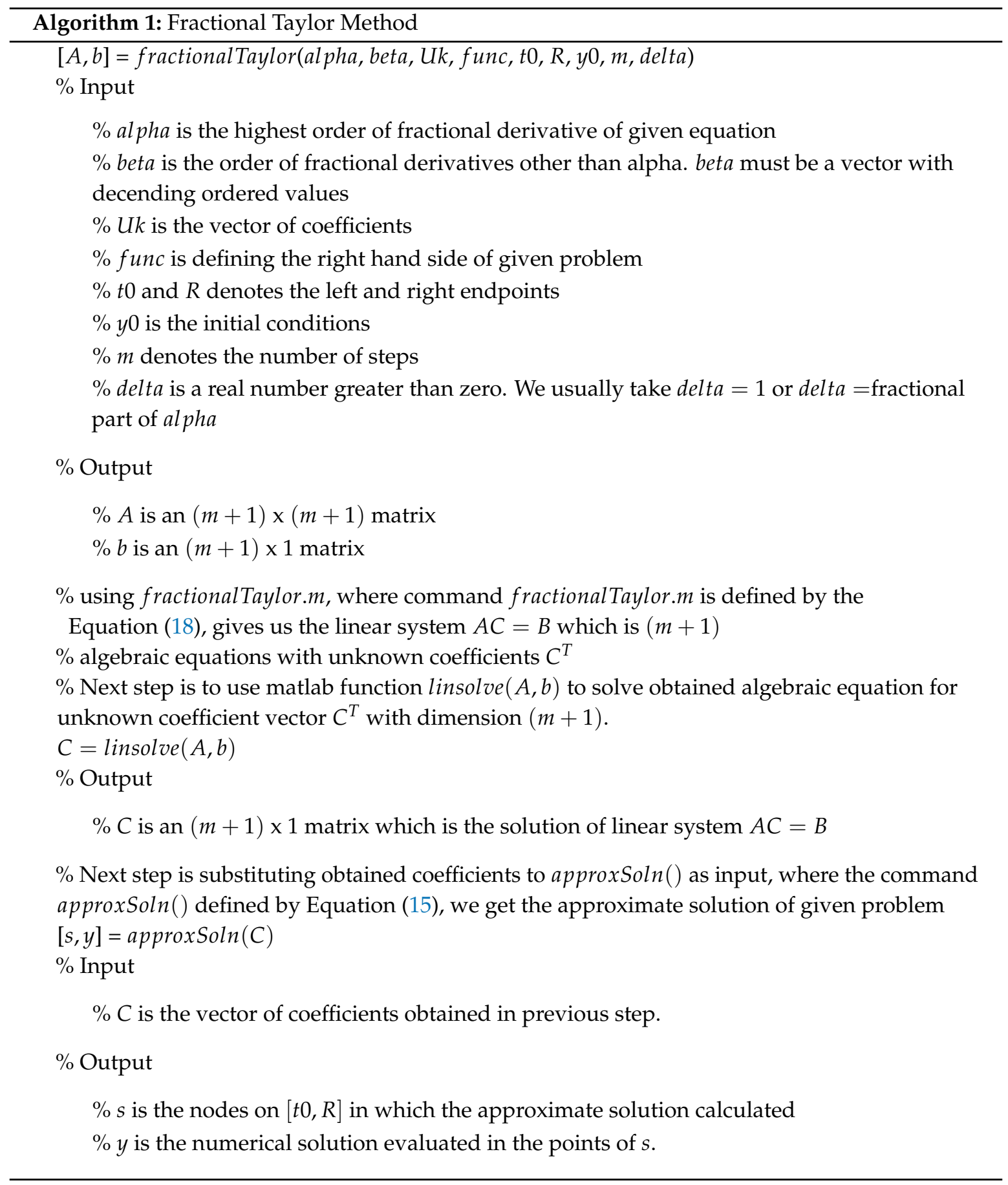

\section{Illustrative Examples}

To illustrate the applicability and effectiveness of the presented method, we give six examples in this section. In each example, we apply the fractional Taylor operational matrix method which is presented in previous section and the approximate results compared with analytical solutions. Obtained results indicate that the proposed technique is very effective for multi-term fractional differential equations. In order to solve the numerical computations, MATLAB version R2015a has been used.

For choosing $\delta$, we usually take either $\delta=1$ or $\delta=\alpha-\lfloor\alpha\rfloor$, the fractional part of $\alpha$. 


\subsection{Example 1}

Consider the following form of multi-order fractional differential equation [59]

$$
\begin{aligned}
D^{\alpha} y(t) & =u_{0} D^{\beta_{0}} y(t)+u_{1} D^{\beta_{1}} y(t)+u_{2} D^{\beta_{2}} y(t)+u_{3} D^{\beta_{3}} y(t)+f(t), 0 \leq t \leq R, \\
y(0) & =V_{0}, \quad y^{\prime}(0)=V_{1}
\end{aligned}
$$

We let $\alpha=2, V_{0}=V_{1}=0, R=1$, the coefficients $u_{0}=u_{2}=-1, u_{1}=2, u_{3}=0$ and $\beta_{0}=0, \beta_{1}=1, \beta_{2}=\frac{1}{2}$ and the function $f(t)$ is

$$
f(t)=t^{7}+\frac{2048}{429 \sqrt{\pi}} t^{6.5}-14 t^{6}+42 t^{5}-t^{2}-\frac{8}{3 \sqrt{\pi}} t^{1.5}+4 t-2 .
$$

where the exact solution is $y(t)=t^{7}-t^{2}$.

We apply the given procedure which is implemented in previous section for solving the Equation (19) step by step.

Firstly, change variable $t \in[0, R]$ to $q \in[0,1]$ by using $q=t / R$.

Now, we use the Equation (8) and get

$$
\frac{1}{R^{\alpha}} D^{\alpha} y_{1}(q)=\frac{u_{0}}{R^{\beta_{0}}} D^{\beta_{0}} y_{1}(q)+\frac{u_{1}}{R^{\beta 1}} D^{\beta_{1}} y_{1}(q)+\frac{u_{2}}{R^{\beta_{2}}} D^{\beta_{2}} y_{1}(q)+\frac{u_{3}}{R^{\beta_{3}}} D^{\beta_{3}} y_{1}(q)+f_{1}(q)
$$

where $0 \leq q \leq 1$.

Next, using Equation (7) we get

$$
\begin{aligned}
\frac{1}{R^{\alpha}}\left(y_{1}(q)-y_{1}(0)-q y_{1} \prime(0)\right) & =\frac{u_{0}}{R^{\beta_{0}}} I^{\alpha-\beta_{0}}\left(y_{1}(q)-y_{1}(0)-q y_{1} \prime(0)\right) \\
& +\frac{u_{1}}{R^{\beta 1}} I^{\alpha-\beta_{1}}\left(y_{1}(q)-y_{1}(0)-q y_{1} \prime(0)\right) \\
& +\frac{u_{2}}{R^{\beta_{2}}} I^{\alpha-\beta_{2}}\left(y_{1}(q)-y_{1}(0)-q y_{1} \prime(0)\right) \\
& +\frac{u_{3}}{R^{\beta_{3}}} I^{\alpha-\beta_{3}}\left(y_{1}(q)-y_{1}(0)-q y_{1} \prime(0)\right) \\
& +I^{\alpha} f_{1}(q) .
\end{aligned}
$$

Now, using Equation (21) and substituting initial conditions $y(0)=V_{0}, y^{\prime}(0)=V_{1}$ into equation

$$
\begin{aligned}
\frac{1}{R^{\alpha}}\left(C^{T} T_{m \delta}(q)-V_{0}-R q V_{1}\right) & =\frac{u_{0}}{R^{\beta_{0}}} I^{\alpha-\beta_{0}}\left(C^{T} T_{m \delta}(q)-V_{0}-R q V_{1}\right) \\
& +\frac{u_{1}}{R^{\beta 1}} I^{\alpha-\beta_{1}}\left(C^{T} T_{m \delta}(q)-V_{0}-R q V_{1}\right) \\
& +\frac{u_{2}}{R^{\beta_{2}}} I^{\alpha-\beta_{2}}\left(C^{T} T_{m \delta}(q)-V_{0}-R q V_{1}\right) \\
& +\frac{u_{3}}{R^{\beta_{3}}} I^{\alpha-\beta_{3}}\left(C^{T} T_{m \delta}(q)-V_{0}-R q V_{1}\right) \\
& +I^{\alpha} f_{1}(q) .
\end{aligned}
$$


From Equation (12), we have

$$
\begin{aligned}
& \frac{1}{R^{\alpha}}\left(C^{T} T_{m \delta}(q)-V_{0}-R q V_{1}\right) \\
& =\frac{u_{0}}{R^{\beta_{0}}} q^{\alpha-\beta_{0}} C^{T}\left(G_{\alpha-\beta_{0}} * T_{m \delta}(q)\right)-\frac{u_{0} q^{\alpha-\beta_{0}}}{R^{\beta_{0}} \Gamma\left(\alpha-\beta_{0}+1\right)} V_{0}-\frac{u_{0} q^{\alpha-\beta_{0}+1}}{R^{\beta_{0}} \Gamma\left(\alpha-\beta_{0}+2\right)} V_{1} \\
& +\frac{u_{1}}{R^{\beta 1}} q^{\alpha-\beta_{1}} C^{T}\left(G_{\alpha-\beta_{1}} * T_{m \delta}(q)\right)-\frac{u_{1} q^{\alpha-\beta_{1}}}{R^{\beta_{1}} \Gamma\left(\alpha-\beta_{1}+1\right)} V_{0}-\frac{u_{1} q^{\alpha-\beta_{1}+1}}{R^{\beta 1} \Gamma\left(\alpha-\beta_{1}+2\right)} V_{1} \\
& +\frac{u_{2}}{R^{\beta_{2}}} q^{\alpha-\beta_{2}} C^{T}\left(G_{\alpha-\beta_{2}} * T_{m \delta}(q)\right)-\frac{u_{2} q^{\alpha-\beta_{2}}}{R^{\beta_{2}} \Gamma\left(\alpha-\beta_{2}+1\right)} V_{0}-\frac{u_{2} q^{\alpha-\beta_{2}+1}}{R^{\beta_{2}} \Gamma\left(\alpha-\beta_{2}+2\right)} V_{1} \\
& +\frac{u_{3}}{R^{\beta_{3}}} q^{\alpha-\beta_{3}} C^{T}\left(G_{\alpha-\beta_{3}} * T_{m \delta}(q)\right)-\frac{u_{3} q^{\alpha-\beta_{3}}}{R^{\beta_{3}} \Gamma\left(\alpha-\beta_{3}+1\right)} V_{0}-\frac{u_{3} s^{\alpha-\beta_{3}+1}}{R^{\beta_{3}} \Gamma\left(\alpha-\beta_{3}+2\right)} V_{1} \\
& +I^{\alpha} f_{1}(q) \text {. }
\end{aligned}
$$

Now, taking $R=1$ in Equation (23) and putting the given values for $V_{0}, V_{1}, u_{i}, \beta_{i}$ where $i=0,1,2,3$ into this equation, we get

$$
C^{T} T_{m \delta}=2 q^{1} C^{T}\left(G_{1} * T_{m \delta}(q)\right)-q^{3 / 2} C^{T}\left(G_{3 / 2} * T_{m \delta}(q)\right)-q^{2} C^{T}\left(G_{1} * T_{m \delta}(q)\right)+I^{2} f_{1}(q)
$$

Finally, taking the collocation points $q_{j}=j / m(j=0,1, \ldots, m)$ generates a linear algebraic system of dimension $m+1$ with unknown vector $C^{T}$. In order to solve this system by using presented method and comparing the results, we choose $\delta=1$ and different values of $m$.

To show the efficiency, we compared the numerical results with the method given in [59].

Table 1, compares the obtained results for absolute error with $m=4,6,7$. We observe from Table 1 that, the absolute errors for presented method are smaller and the numerical solution is more accurate for the same size of $m$.

Table 1. The comparison absolute errors of the present scheme and method given in [59] with $m=4,6,7$.

\begin{tabular}{ccccccc}
\hline & Present Method & Method in [59] & Present Method & Method in [59] & Present Method & Method in [59] \\
\cline { 2 - 7 } & $\boldsymbol{m}=\mathbf{4}$ & $\boldsymbol{m}=\mathbf{4}$ & $\boldsymbol{m}=\mathbf{6}$ & $\boldsymbol{m}=\mathbf{6}$ & $\boldsymbol{m}=\mathbf{7}$ & $\boldsymbol{m}=\mathbf{7}$ \\
\hline 0.2 & 0.0116 & 0.0844 & $6.81430698097618 \times 10^{-7}$ & 0.0044 & $1.040834086 \times 10^{-16}$ & $2.81025203108243 \times 10^{-15}$ \\
0.4 & 0.0032 & 0.3501 & $1.01100805164899 \times 10^{-4}$ & 0.0079 & $2.498001805 \times 10^{-16}$ & $6.63358257213531 \times 10^{-15}$ \\
0.6 & 0.0108 & 0.6734 & $1.2907314422994 \times 10^{-5}$ & 0.0143 & $1.665334537 \times 10^{-16}$ & $3.27515792264421 \times 10^{-15}$ \\
0.8 & 0.0037 & 1.0234 & $1.16246682382747 \times 10^{-4}$ & 0.0214 & $3.330669074 \times 10^{-16}$ & $4.25770529943748 \times 10^{-14}$ \\
1.0 & 0.0026 & 1.6700 & $1.11299947542775 \times 10^{-5}$ & 0.0280 & $1.110223025 \times 10^{-16}$ & $2.43819897540083 \times 10^{-13}$ \\
\hline
\end{tabular}

In Figures 1-3, we present the graphical representation of comparison between exact solution and the numerical solutions obtained by proposed method and the method of [59] for the problem (19) with $m=4,6,7$ respectively. From these results, we can conclude that $m=4$ and $m=6$ give larger absolute error, while $m=7$ gives smaller absolute error $\left(10^{-16}\right)$ and more precise numerical solution. These comparisons also shows that the results obtained by proposed method is closer to the exact solution than the results of [59]. 


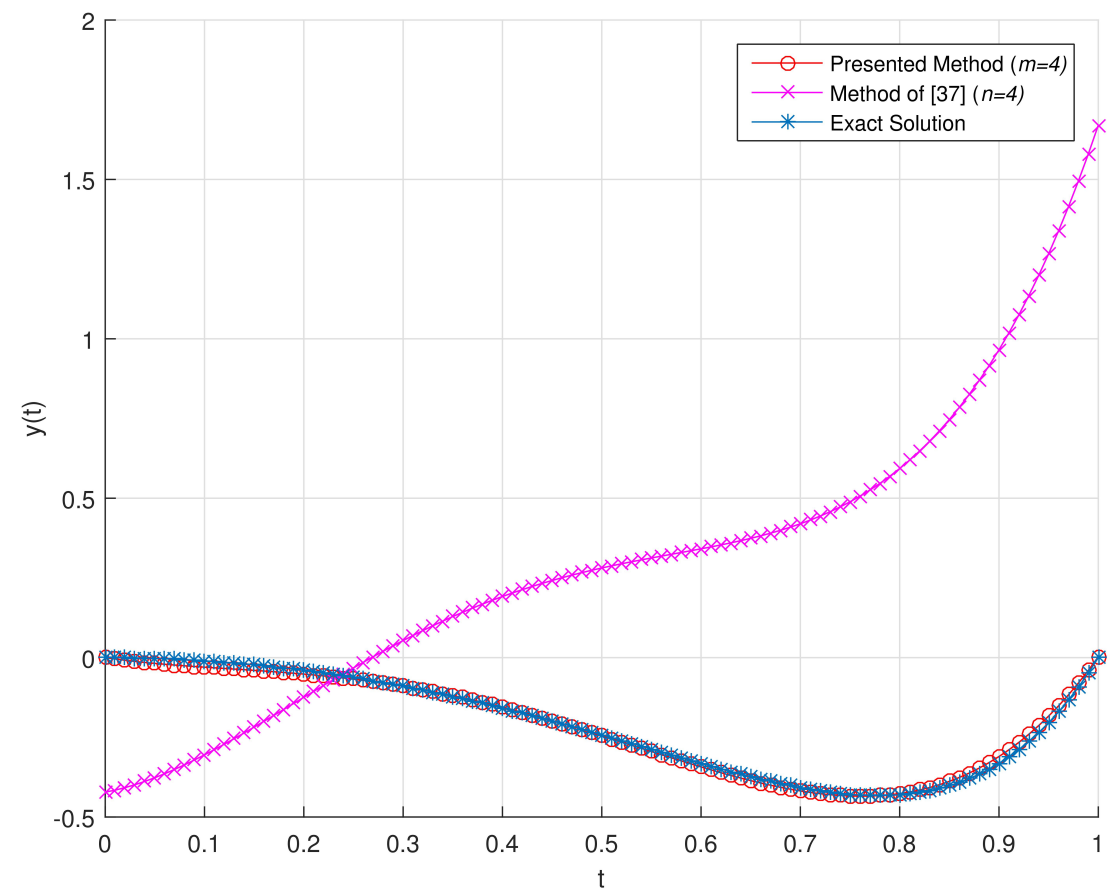

Figure 1. The comparison between exact solution and the numerical solutions obtained by proposed method and the method of [59] with $m, n=4$.

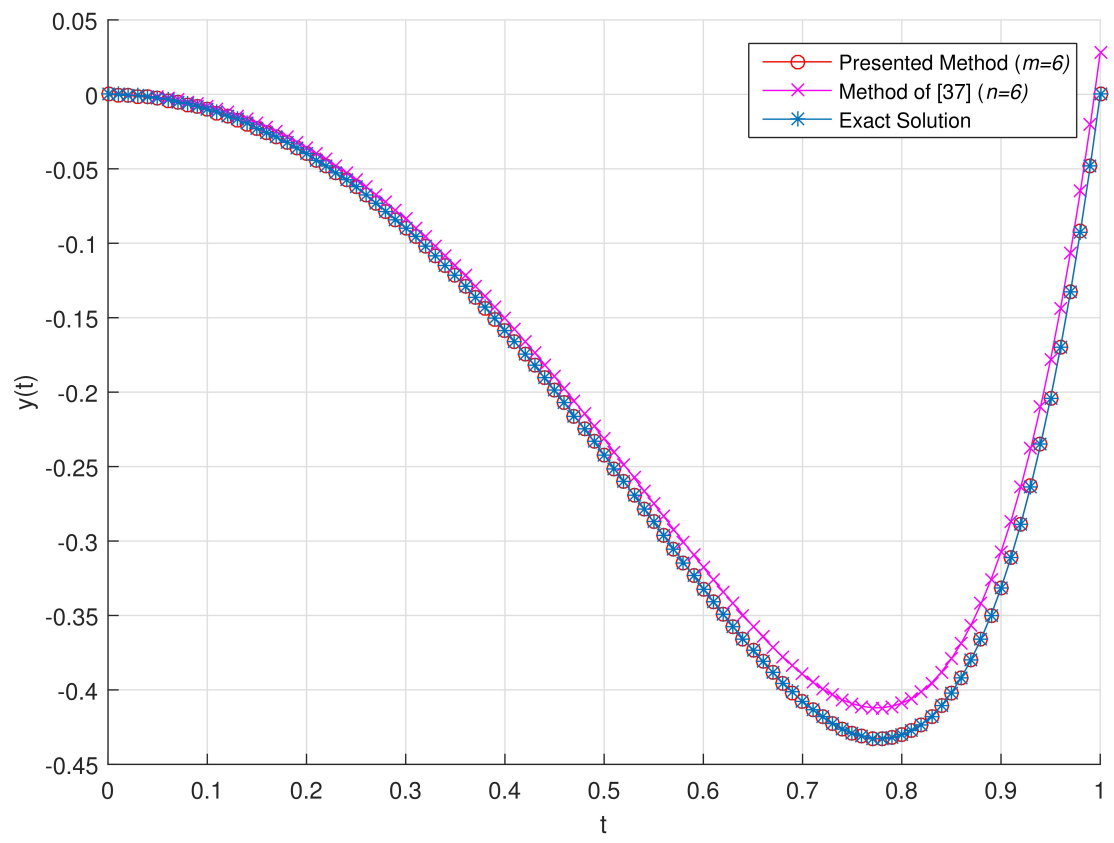

Figure 2. The comparison between exact solution and the numerical solutions obtained by proposed method and the method of [59] with $m, n=6$. 


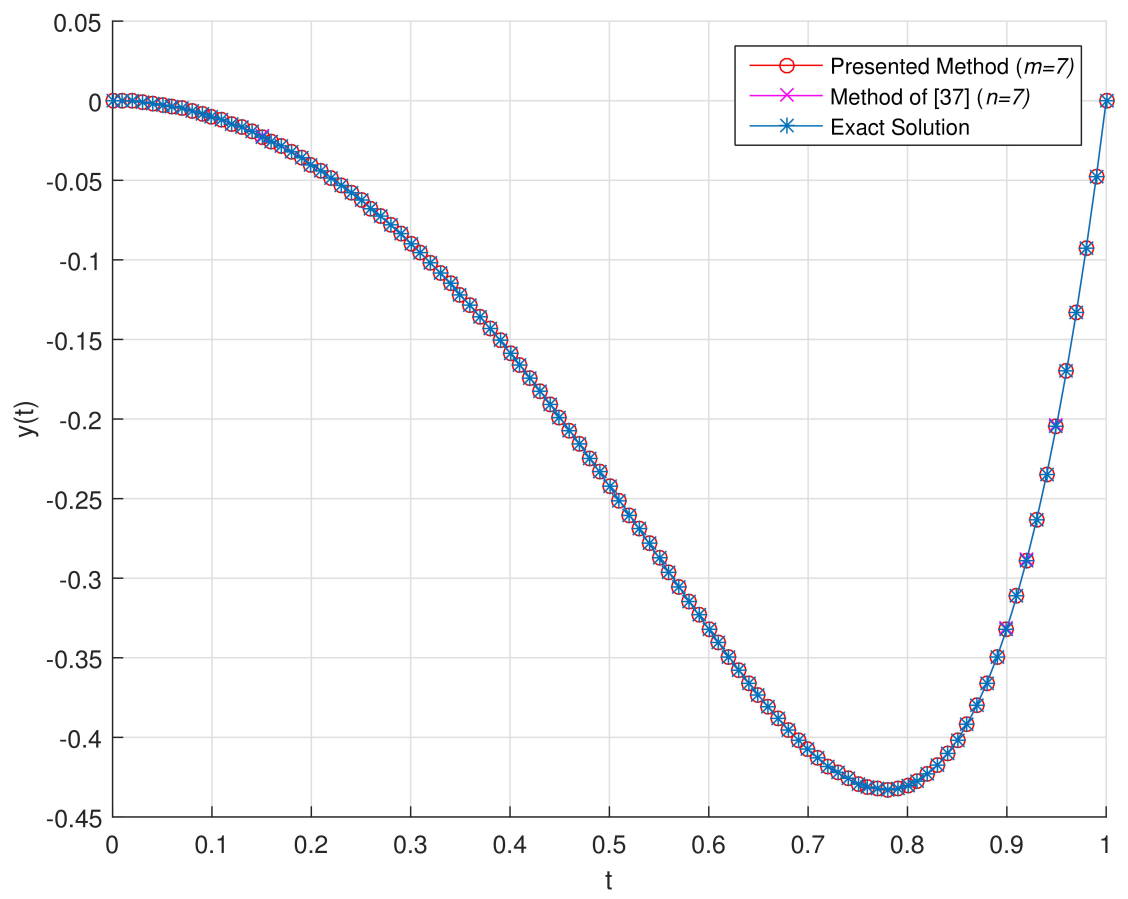

Figure 3. The comparison between exact solution and the numerical solutions obtained by proposed method and the method of [59] with $m, n=7$.

In Figure 4, we show the graphical representation of absolute errors obtained by using proposed method and the method of [59] with $m, n=6$.

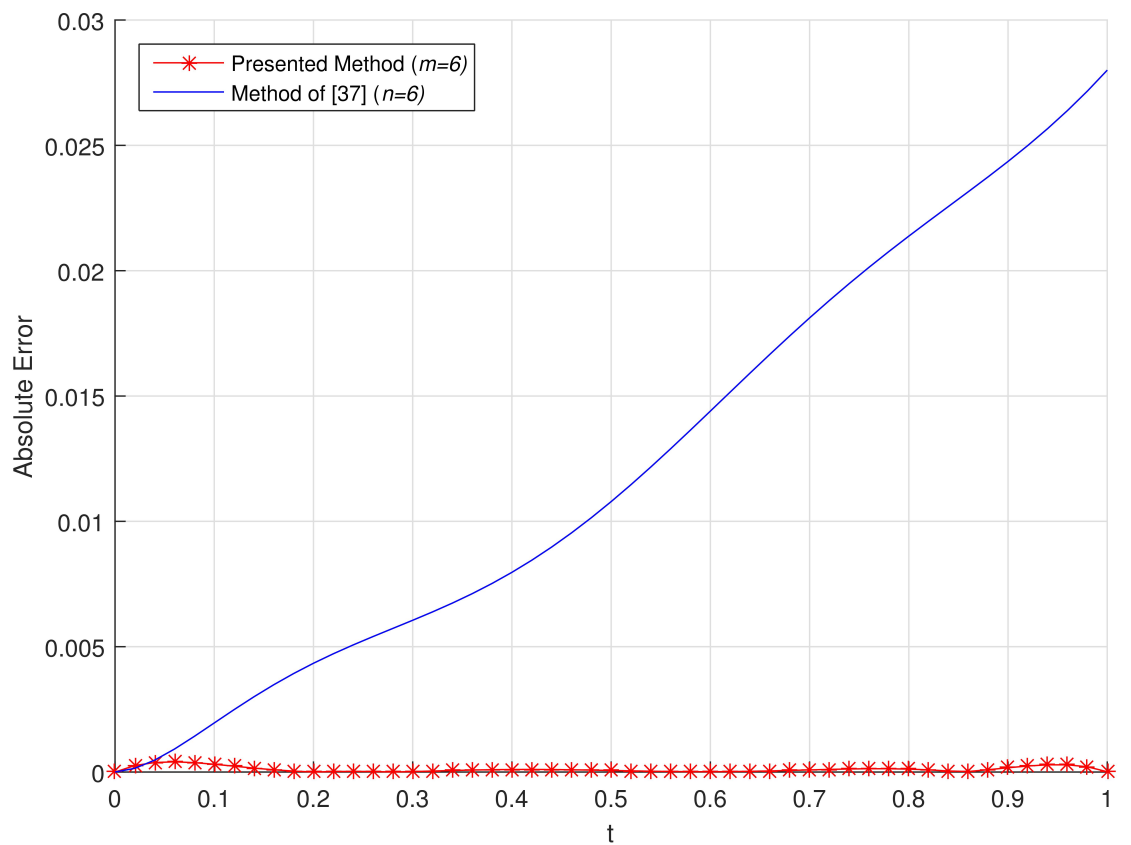

Figure 4. The behaviour of absolute errors obtained by using proposed method and the method of [59] with $m, n=6$. 
From Figure 4, we can conclude that the absolute error obtained by our method is remaining smaller and stable while the absolute error of other method is increasing in the interval $[0,1]$.

In Figures 5 and 6, we give the graphical representation of absolute errors obtained by using proposed method with $m=4,7$ respectively.

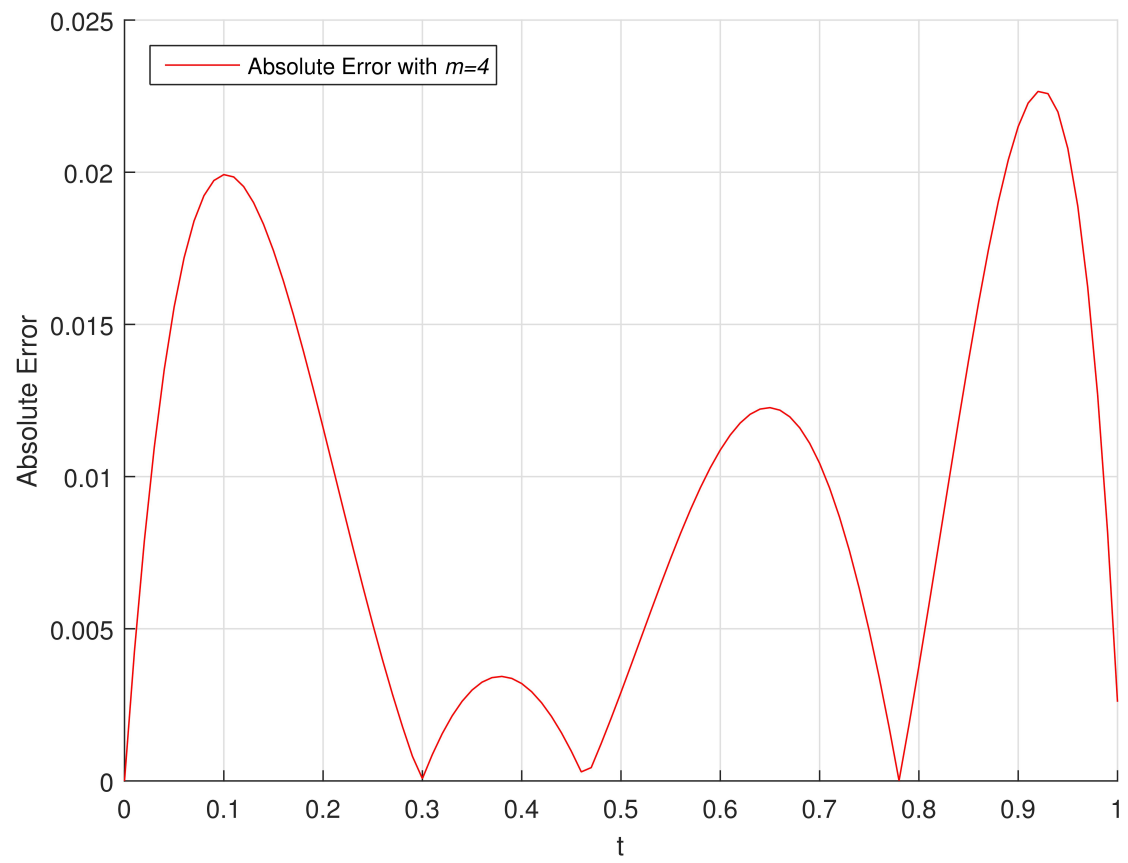

Figure 5. The absolute error with $m=4$.

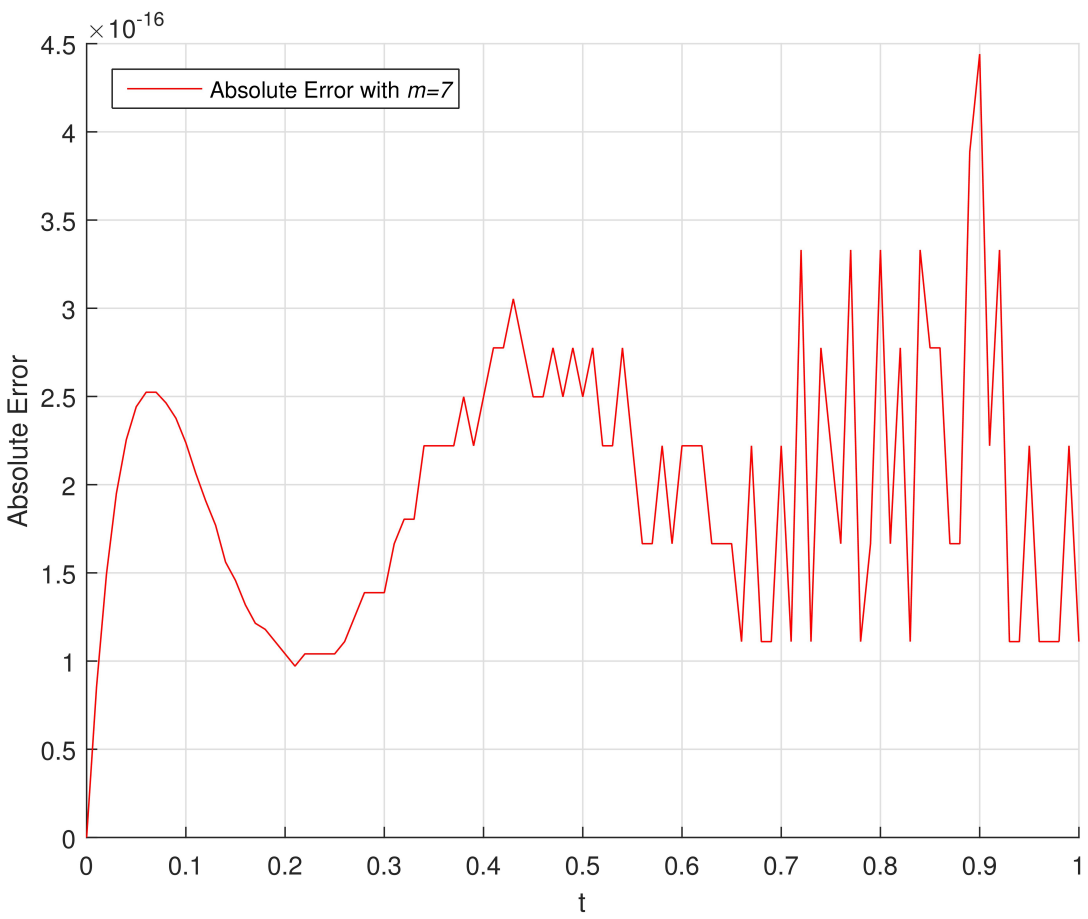

Figure 6. The absolute error with $m=7$.

A pseudo-code for MATLAB implementation of Example 1 is given in Algorithm 2 below : 


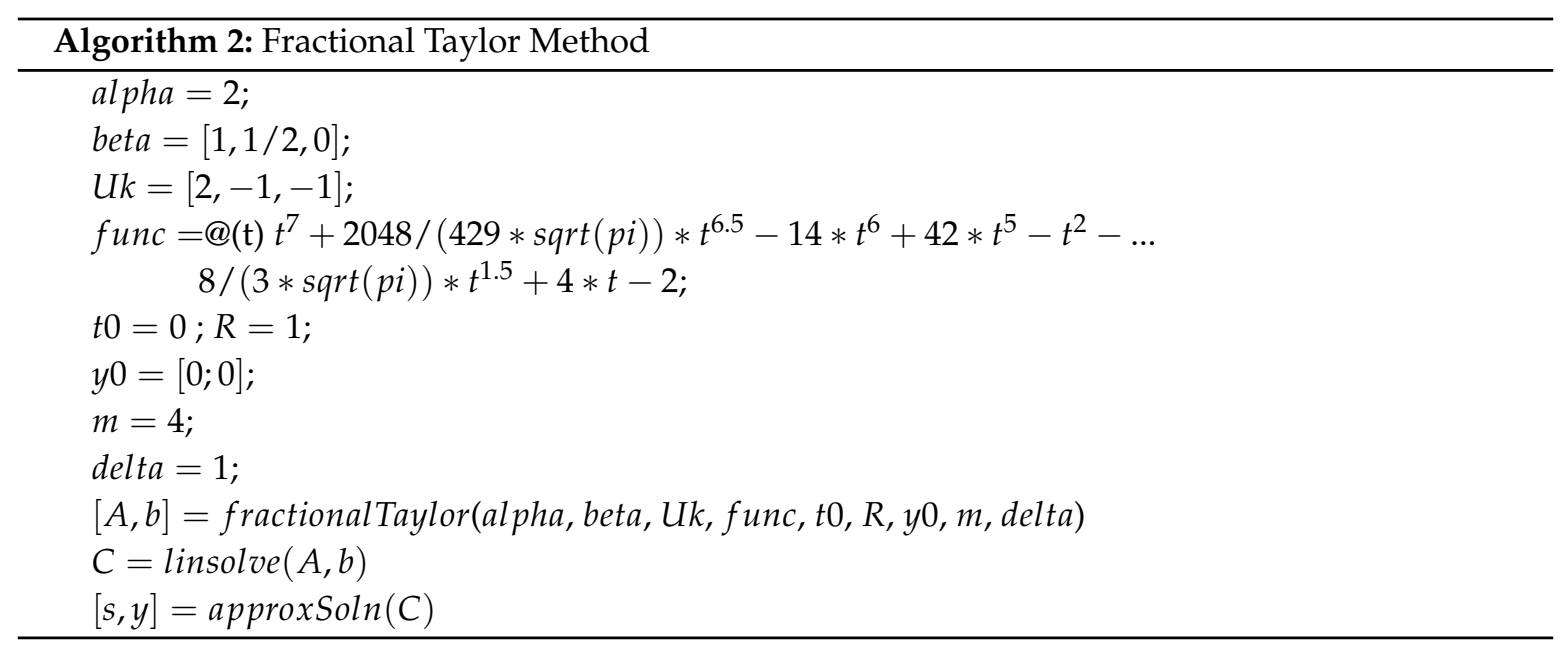

\subsection{Example 2}

In this example, we consider the Equation (19) with $\alpha=2, V_{0}=V_{1}=0$, the coefficients $u_{0}=$ $u_{2}=-1, u_{1}=0, u_{3}=2$ and $\beta_{0}=0, \beta_{2}=\frac{2}{3}, \beta_{3}=\frac{5}{3}$ and the function is

$$
f(t)=t^{3}+6 t-\frac{12}{\Gamma\left(\frac{7}{3}\right)} t^{\frac{4}{3}}+\frac{6}{\Gamma\left(\frac{10}{3}\right)} t^{\frac{7}{3}} .
$$

The exact solution of this equation is $y(t)=t^{3}$ [59].

Applying the same procedure to given problem as presented in Example 1, we get the following equation

$$
C^{T} T_{m \delta}=2 q^{1 / 3} C^{T}\left(G_{1 / 3} * T_{m \delta}(q)\right)-q^{4 / 3} C^{T}\left(G_{4 / 3} * T_{m \delta}(q)\right)-q^{2} C^{T}\left(G_{2} * T_{m \delta}(q)\right)+I^{2} f_{1}(q)
$$

As we stated in previous example, collocating this equation at the nodes $q_{j}=j / m(j=0,1, \ldots, m)$ generates a system of algebraic equations. In this example, to solve this sysem for $C^{T}$, we choose $\delta=1,1.5$ and different values of $m$.

Table 2 shows the results for obtained absolute errors by using presented method with $m=2,3$. From these results, we can see that, there is satisfactory agreement between the exact solution and numerical solutions. The absolute error is achieved about $10^{-15}$. We also note that, the proposed method gives better results for $m=2$ by taking $\delta=1.5$.

Table 2. The absolute errors with $m=2,3$.

\begin{tabular}{cccc}
\hline $\boldsymbol{t}$ & $\delta=\mathbf{1}, \boldsymbol{m = 2}$ & $\boldsymbol{\delta}=\mathbf{1 . 5}, \boldsymbol{m = 2}$ & $\delta=\mathbf{1}, \boldsymbol{m = 3}$ \\
\hline 0 & 0 & 0 & 0 \\
0.1 & 0.010209105 & $1.3 \times 10^{-17}$ & $7.42 \times 10^{-17}$ \\
0.2 & 0.008778787 & $4.68 \times 10^{-17}$ & $1.232 \times 10^{-16}$ \\
0.3 & 0.001709047 & $1.11 \times 10^{-16}$ & $1.769 \times 10^{-16}$ \\
0.4 & 0.005000117 & $2.082 \times 10^{-16}$ & $2.637 \times 10^{-16}$ \\
0.5 & 0.005348703 & $3.608 \times 10^{-16}$ & $4.163 \times 10^{-16}$ \\
0.6 & 0.006663287 & $5.829 \times 10^{-16}$ & $6.661 \times 10^{-16}$ \\
0.7 & 0.037035855 & $8.882 \times 10^{-16}$ & $9.992 \times 10^{-16}$ \\
0.8 & 0.091769001 & $1.2212 \times 10^{-15}$ & $1.5543 \times 10^{-15}$ \\
0.9 & 0.176862723 & $1.6653 \times 10^{-15}$ & $1.9984 \times 10^{-15}$ \\
1.0 & 0.2983170221 & $2.2204 \times 10^{-15}$ & $2.8866 \times 10^{-15}$ \\
\hline
\end{tabular}


In Figure 7a, we show the graphical representation of obtained numerical solution and the exact solution of the given problem. Figure $7 \mathrm{~b}$ presents the obtained absolute error by using proposed method with $m=3$.

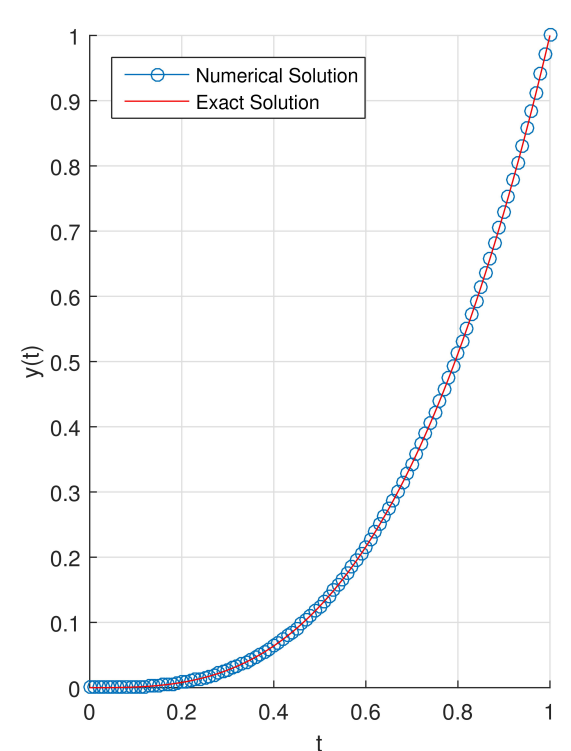

(a)

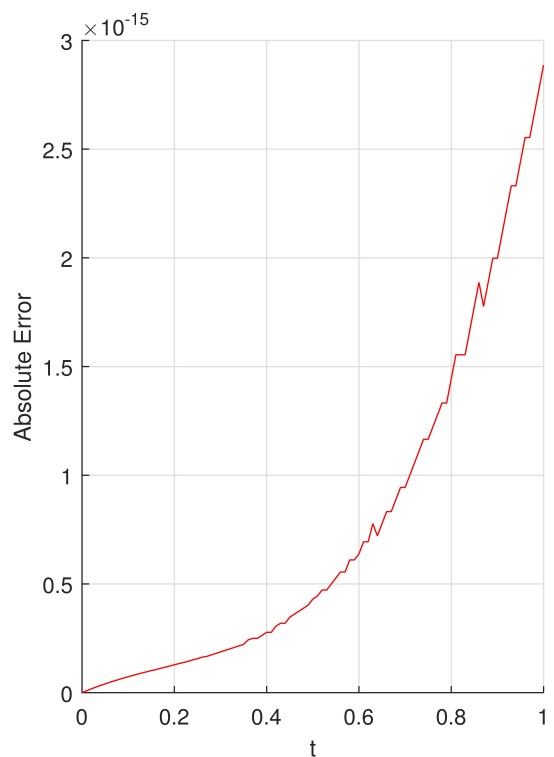

(b)

Figure 7. (a) The numerical and the exact solutions with $m=3$. (b) The absolute error with $m=3$.

\subsection{Example 3}

Consider the multi-term fractional order initial value problem [54]

$$
D^{(2.2)} y(t)+1.3 D^{(1.5)} y(t)+2.6 y(t)=\sin (2 t),
$$

with initial conditions

$$
y(0)=y \prime(0)=y^{\prime \prime}(0)=0,
$$

where the equation have the series solution given by [52]

$$
\begin{aligned}
y_{s}(t) & =\frac{28561}{3600000} t^{6}+\frac{2}{\Gamma(4.2)} t^{3.2}-\frac{13}{5 \Gamma(4.9)} t^{3.9}+\frac{169}{50 \Gamma(5.6)} t^{4.6} \\
& -\frac{8}{\Gamma(6.2)} t^{5.2}-\frac{2197}{500 \Gamma(6.3)} t^{5.3}-\frac{26}{5 \Gamma(6.4)} t^{5.4}+\frac{52}{5 \Gamma(6.9)} t^{5.9} .
\end{aligned}
$$

In order to solve this problem, we choose $\delta=1$, and $m=10$.

We give the comparison of series solution and the numerical solution obtained by presented method in Table 3. Table 4 compares the obtained absolute errors by using presented method with the results of [54]. From this compared results, it can be seen that the approximate solution is very close to series solution for a small number of $m$ for the given method.

From the compared results of Table 4, we can conclude that the proposed method has better approach to series solution with a smaller $m$. 
Table 3. Comparison of numerical solution with series solution for Example 3.

\begin{tabular}{ccc}
\hline$t$ & Series Solution [52] & Present Method $\boldsymbol{m = 1 0}$ \\
\hline 0.0 & 0 & 0 \\
0.1 & 0.000147766 & 0.000147731 \\
0.2 & 0.001274983 & 0.001275552 \\
0.3 & 0.00439917 & 0.00440567 \\
0.4 & 0.010405758 & 0.010441315 \\
0.5 & 0.019962077 & 0.020094648 \\
0.6 & 0.033452511 & 0.033841301 \\
0.7 & 0.050923716 & 0.051890573 \\
0.8 & 0.0720381 & 0.074169634 \\
0.9 & 0.096035415 & 0.100321388 \\
\hline
\end{tabular}

Table 4. Comparison of absolute errors for Example 3.

\begin{tabular}{ccc}
\hline $\boldsymbol{t}$ & Present Method $\boldsymbol{m}=\mathbf{1 0}$ & Method in [54] $\boldsymbol{m = \mathbf { 2 0 }}$ \\
\hline 0.0 & 0 & 0 \\
0.1 & $3.47449 \times 10^{-8}$ & $5.2560 \times 10^{-7}$ \\
0.2 & $5.69366 \times 10^{-7}$ & $1.7150 \times 10^{-6}$ \\
0.3 & $6.49968 \times 10^{-6}$ & $8.2260 \times 10^{-6}$ \\
0.4 & $3.55576 \times 10^{-5}$ & $3.7820 \times 10^{-5}$ \\
0.5 & 0.000132571 & 0.0001353 \\
0.6 & 0.00038879 & 0.000392 \\
0.7 & 0.000966858 & 0.0009704 \\
0.8 & 0.002131534 & 0.002135 \\
0.9 & 0.004285973 & 0.00429 \\
\hline
\end{tabular}

The graphical representation of comparison between series solution and numerical solutions obtained by presented method and the method of [54] in the interval [0,1] is illustrated in Figure 8.

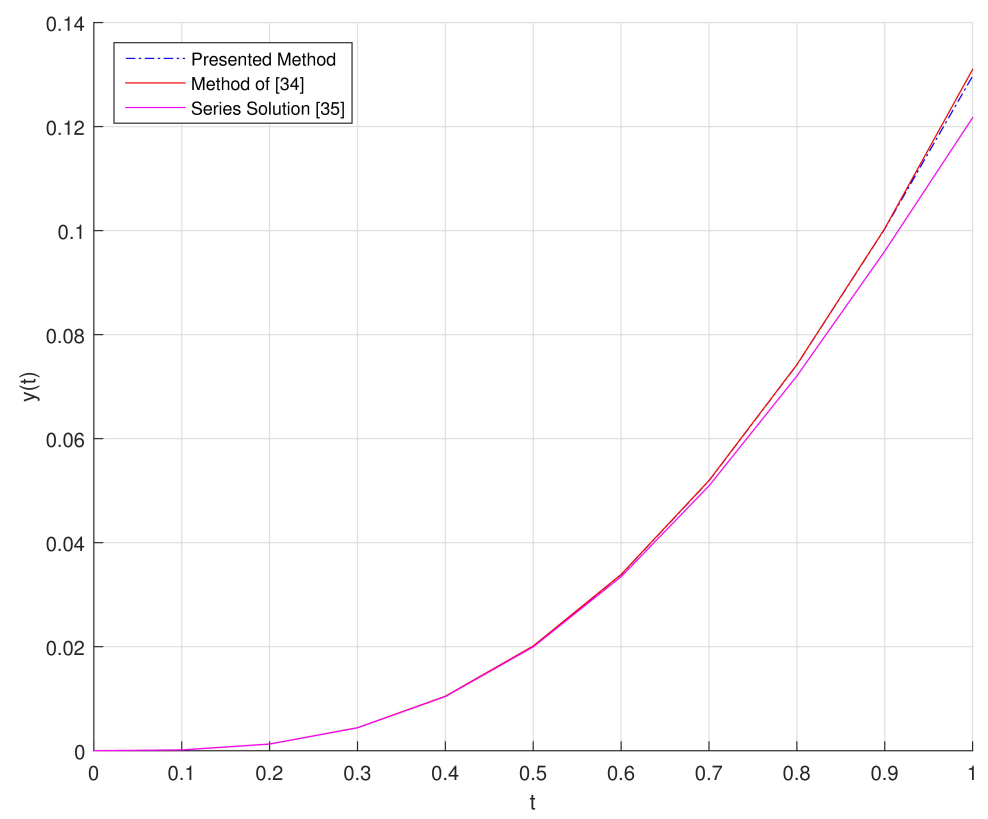

Figure 8. The comparison between series solution and numerical solutions obtained by proposed method and the method of [54] with $m=10$. 
In Figure 9, we show present graphical representation of absolute errors obtained by using proposed method and the method of [54] with $m=10$.

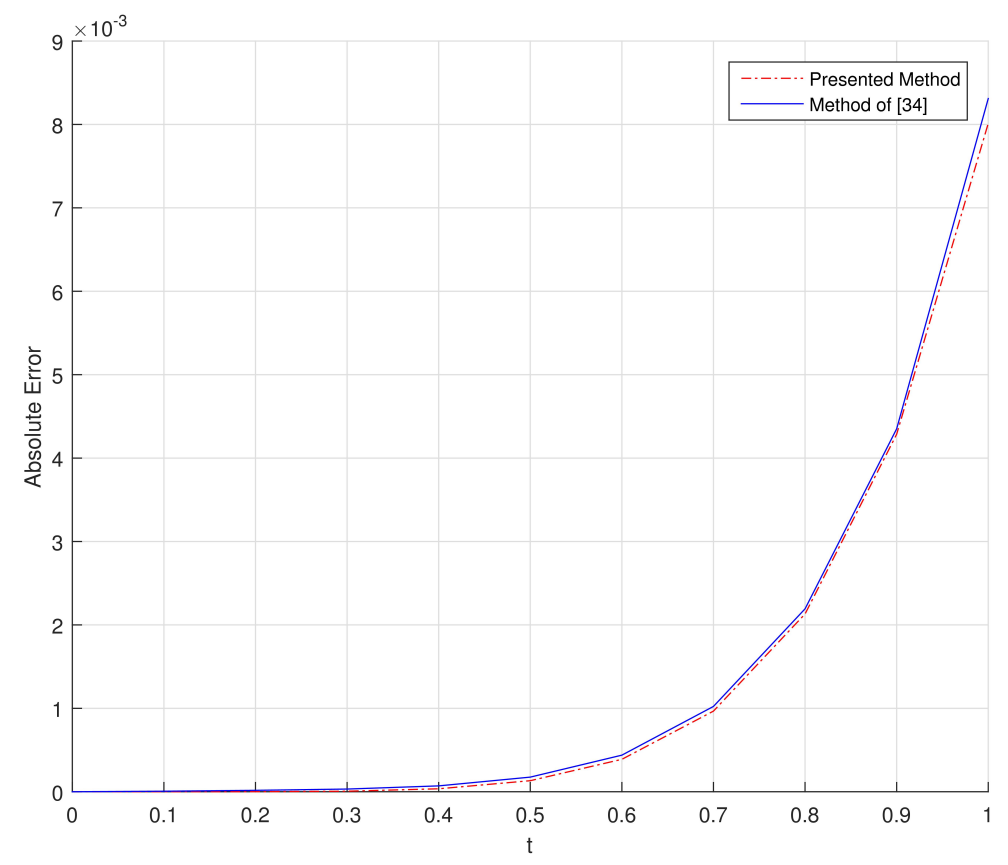

Figure 9. The behaviour of absolute errors obtained by using proposed method and the method of [54].

In Figure 10, we show the graphical representation for series solution and the numerical results of presented method for the interval $[0,10]$. The results plotted in Figure 10 are in a very good and satisfactory agreement with the series solution given in [52] and the results of [60].

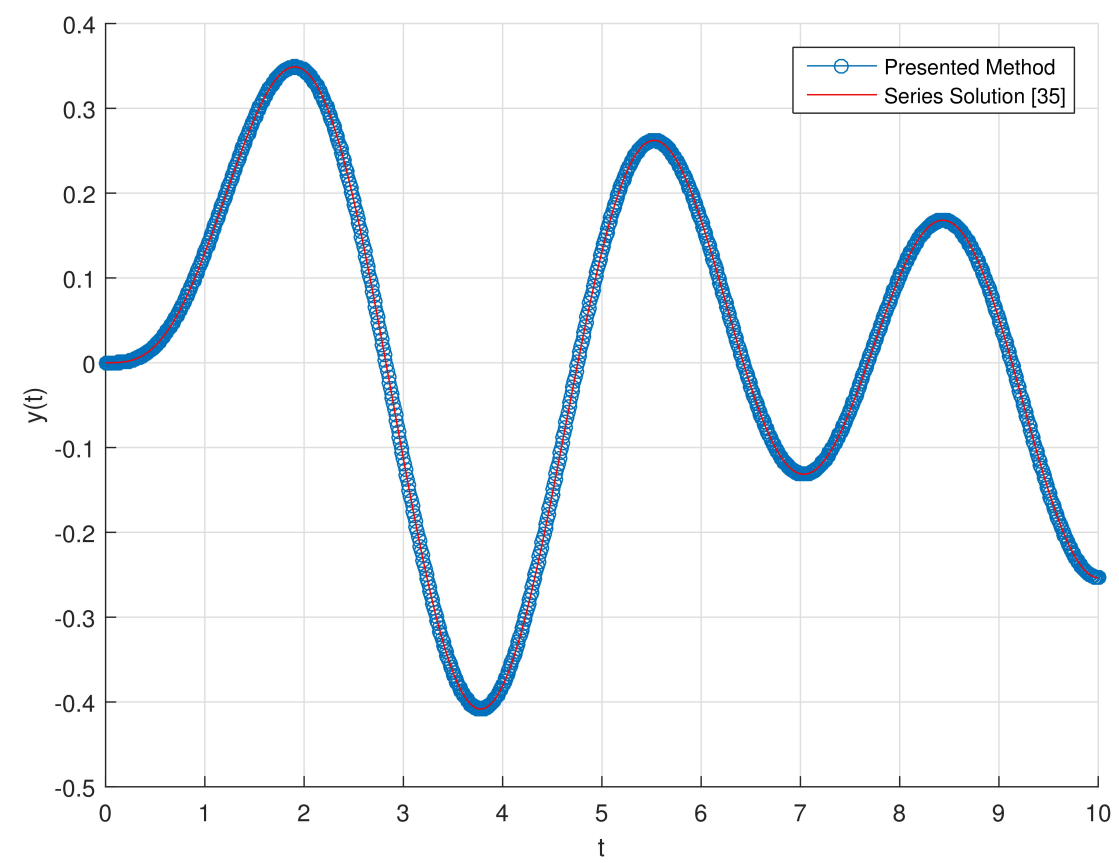

Figure 10. The behaviour of series solution and the numerical solution obtained by proposed method for the interval $[0,10]$. 


\subsection{Example 4}

Motivated by [50], we consider the following form of fractional differential equation,

$$
D^{\alpha} y(t)+y(t)= \begin{cases}\frac{2}{\Gamma(3-\alpha)} t^{2-\alpha}+t^{2}-t, & \alpha>1 \\ \frac{2}{\Gamma(3-\alpha)} t^{2-\alpha}-\frac{1}{\Gamma(2-\alpha)} t^{1-\alpha}+t^{2}-t, & \alpha \leq 1\end{cases}
$$

with initial conditions

$$
y(0)=0, y^{\prime}(0)=-1
$$

whose exact solution is $y(t)=t^{2}-t$.

In order to apply the presented method to Equation (28) and compare the results with methods of $[54,61,62]$, we solve this problem with $\alpha=0.3,0.5,0.7,1.25,1.5,1.85$, and different values for $\delta$ and $m$. The obtained results are presented as below.

In Table 5, we list the results of obtained absolute errors for $\alpha=0.3,0.5,0.7$ by use of presented method. Also, the results for $\alpha=1.25,1.5,1.85$ are given in Table 6 .

Table 5. The absolute errors with $m=3$ and $\alpha<1$ for Example 4.

\begin{tabular}{cccc}
\hline $\boldsymbol{t}$ & $\boldsymbol{\alpha}=\mathbf{0 . 3}$ & $\boldsymbol{\alpha}=\mathbf{0 . 5}$ & $\boldsymbol{\alpha}=\mathbf{0 . 7}$ \\
\hline 0 & 0 & 0 & 0 \\
0.1 & $4.16 \times 10^{-17}$ & $8.33 \times 10^{-17}$ & $1.94 \times 10^{-16}$ \\
0.2 & $8.33 \times 10^{-17}$ & $5.55 \times 10^{-17}$ & $2.78 \times 10^{-16}$ \\
0.3 & $1.11 \times 10^{-16}$ & $2.78 \times 10^{-17}$ & $2.50 \times 10^{-16}$ \\
0.4 & $1.67 \times 10^{-16}$ & $1.39 \times 10^{-16}$ & $2.50 \times 10^{-16}$ \\
0.5 & $1.67 \times 10^{-16}$ & $1.11 \times 10^{-16}$ & $1.67 \times 10^{-16}$ \\
0.6 & $1.67 \times 10^{-16}$ & $5.55 \times 10^{-17}$ & $2.78 \times 10^{-17}$ \\
0.7 & $1.67 \times 10^{-16}$ & $8.33 \times 10^{-17}$ & $8.33 \times 10^{-17}$ \\
0.8 & $3.05 \times 10^{-16}$ & $5.55 \times 10^{-17}$ & $1.11 \times 10^{-16}$ \\
0.9 & $2.08 \times 10^{-16}$ & $1.25 \times 10^{-16}$ & $1.39 \times 10^{-16}$ \\
1.0 & $1.91 \times 10^{-16}$ & $1.26 \times 10^{-16}$ & $8.91 \times 10^{-17}$ \\
\hline
\end{tabular}

Table 6. The absolute errors with $m=3$ and $\alpha>1$ for Example 4.

\begin{tabular}{cccc}
\hline$t$ & $\alpha=\mathbf{1 . 2 5}$ & $\alpha=\mathbf{1 . 5}$ & $\alpha=\mathbf{1 . 8 5}$ \\
\hline 0.0 & 0 & 0 & 0 \\
0.1 & $1.39 \times 10^{-17}$ & $2.78 \times 10^{-17}$ & $1.25 \times 10^{-16}$ \\
0.2 & $5.55 \times 10^{-17}$ & $5.55 \times 10^{-17}$ & $1.94 \times 10^{-16}$ \\
0.3 & $5.55 \times 10^{-17}$ & $5.55 \times 10^{-17}$ & $2.22 \times 10^{-16}$ \\
0.4 & $5.55 \times 10^{-17}$ & $2.78 \times 10^{-17}$ & $2.50 \times 10^{-16}$ \\
0.5 & $1.11 \times 10^{-16}$ & 0 & $2.22 \times 10^{-16}$ \\
0.6 & $1.67 \times 10^{-16}$ & $5.55 \times 10^{-17}$ & $1.67 \times 10^{-16}$ \\
0.7 & $1.94 \times 10^{-16}$ & $5.55 \times 10^{-17}$ & $5.55 \times 10^{-17}$ \\
0.8 & $3.05 \times 10^{-16}$ & $1.39 \times 10^{-16}$ & $5.55 \times 10^{-17}$ \\
0.9 & $1.11 \times 10^{-16}$ & $8.33 \times 10^{-17}$ & $1.39 \times 10^{-17}$ \\
1.0 & $8.21 \times 10^{-17}$ & $1.97 \times 10^{-16}$ & $1.06 \times 10^{-16}$ \\
\hline
\end{tabular}

In Figure 11a,b, we present the graphical representation of obtained results for numerical and exact solution of the given problem and absolute error for $\alpha=1.5$ in the interval $[0,1]$.

In Figure 12, we plot the graphical representation for behavior of the obtained numerical solution by use of the presented method and the exact solution of the given problem for $\alpha=1.5$ in the interval $[0,15]$. 

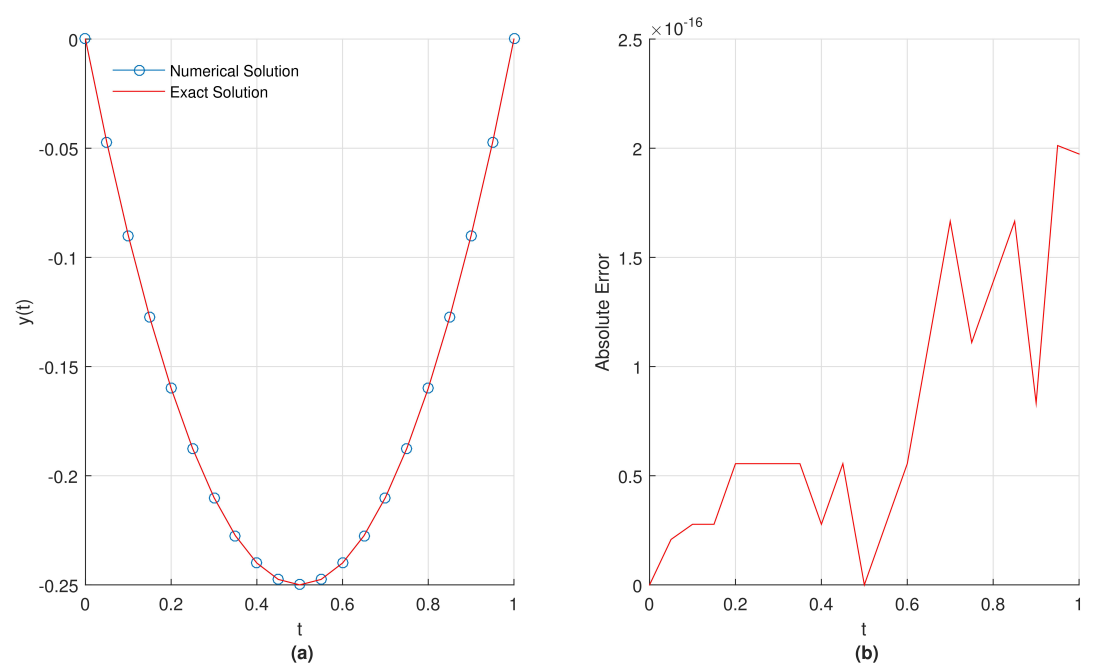

Figure 11. (a) The numerical and exact solutions for $\alpha=1.5$. (b) The absolute error for $\alpha=1.5$.

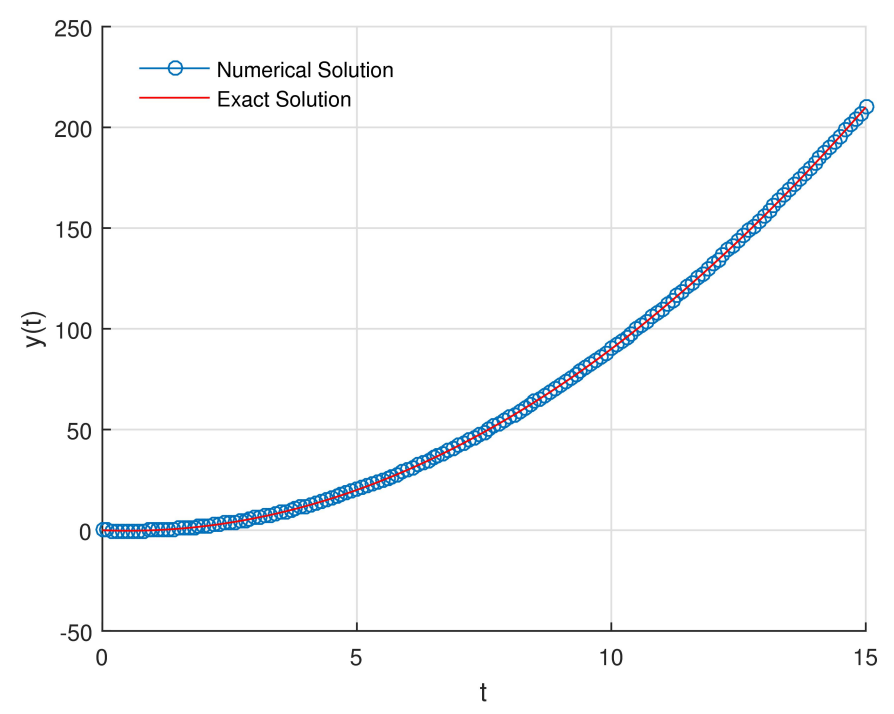

Figure 12. The behaviour of the obtained numerical and exact solutions with $\alpha=1.5$ for the interval $t \in[0,15]$.

Table 7 lists the obtained absolute errors for the given problem (28) at $t=1,5,10,50$ and $\alpha=1.5$ by use of presented method and some other methods in literature [54,61,62]. From this compared results, we can say that the numerical solution obtained by use of proposed method is in better agreement with the exact solution and obtained absolute error is smaller.

Table 7. Comparison of absolute errors between proposed method and some other numerical methods in literature at $t=1,5,10,50$ for $\alpha=1.5$.

\begin{tabular}{ccccc}
\hline \multirow{t}{*}{} & Presented Method & Method of [63] & Method of [50] & Method of [64] \\
\cline { 2 - 5 } & $\boldsymbol{\delta}=\mathbf{1 / 2 , \boldsymbol { m } = \mathbf { 4 }}$ & $\boldsymbol{n}=\mathbf{2 0}$ & $\boldsymbol{h}=\mathbf{1 / 3 2 0}$ & $\boldsymbol{p}=\mathbf{1}, \boldsymbol{T}=\mathbf{1}$ \\
\hline 1 & $7.99361 \times 10^{-14}$ & $9.10 \times 10^{-5}$ & $3.42 \times 10^{-3}$ & - \\
5 & $2.55795 \times 10^{-13}$ & $2.42 \times 10^{-3}$ & - & - \\
10 & $1.42109 \times 10^{-13}$ & $5.50 \times 10^{-3}$ & - & - \\
50 & $3.63798 \times 10^{-12}$ & $3.74 \times 10^{-2}$ & - & 1.2 \\
\hline
\end{tabular}


In Figure 13, the behaviour of absolute error for $\alpha=1.5$ with $m=4$ and $\delta=1 / 2,1$ at $t \in[0,50]$ is presented. From this graph, it can be seen that we get better results by taking $\delta=1 / 2$ for this example and the numerical solution is very close to exact solution for a small number of $m$.

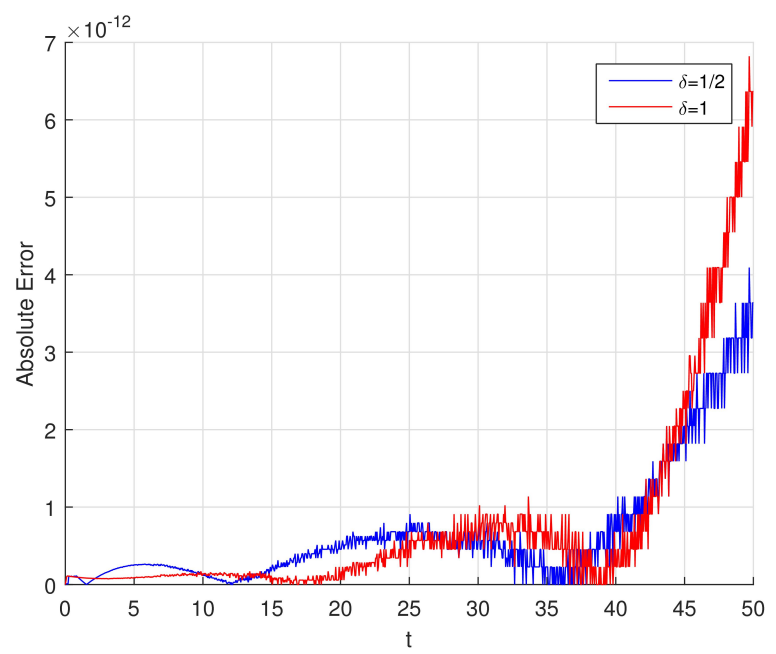

Figure 13. The behaviour of the absolute errors for proposed method where $\alpha=1.5, t \in[0,50]$ with $m=4$ and $\delta=1 / 2,1$.

\subsection{Example 5}

In this example, we consider the following form of linear multi-term fractional differential equation with variable coefficients [65]

$$
a D^{2} y(t)+b(t) D^{\beta_{1}} y(t)+c(t) D y(t)+e(t) D^{\beta_{2}} y(t)+k(t) y(t)=f(t),
$$

with,

$$
y(0)=2, y \prime(0)=0
$$

where $0<\beta_{2}<1,1<\beta_{1}<2$ and

$$
f(t)=-a-\frac{b(t)}{\Gamma\left(3-\beta_{1}\right)} t^{2-\beta_{1}}-c(t) t-\frac{e(t)}{\Gamma\left(3-\beta_{2}\right)} t^{2-\beta_{2}}+k(t)\left(2-\frac{t^{2}}{2}\right)
$$

whose the exact solution is given by $y(t)=2-\frac{t^{2}}{2}$.

We give the numerical solution for the given problem by proposed method for $a=1, b(t)=$ $\sqrt{t}, c(t)=t^{\frac{1}{3}}, e(t)=t^{\frac{1}{4}}, k(t)=t^{\frac{1}{5}}, \beta_{2}=0.333, \beta_{1}=1.234$ with $\delta=1$.

In Table 8, we give the results for maximum errors obtained by use of proposed method and comparison with the results of $[65,66]$. From this compared results, we can see that the numerical solution obtained by use of proposed method is closer to the exact solution.

Table 8. Maximum errors of Example 5 for $R=1$ with $m=3,4,5,6,10,20,40$.

\begin{tabular}{cccc}
\hline$m$ & Present Method & Method Given in [66] & Method Given in [65] \\
\hline 3 & $4.44089 \times 10^{-16}$ & $4.4409 \times 10^{-16}$ & - \\
4 & $6.66134 \times 10^{-16}$ & $1.4633 \times 10^{-13}$ & - \\
5 & $4.44089 \times 10^{-16}$ & $3.2743 \times 10^{-12}$ & $6.88384 \times 10^{-5}$ \\
6 & $4.44089 \times 10^{-16}$ & $1.0725 \times 10^{-13}$ & - \\
10 & $2.22045 \times 10^{-15}$ & - & $3.00351 \times 10^{-6}$ \\
20 & $3.47278 \times 10^{-13}$ & - & $1.67837 \times 10^{-7}$ \\
40 & $1.46549 \times 10^{-13}$ & - & $1.02241 \times 10^{-8}$ \\
\hline
\end{tabular}


Figure 14 presents the graphical representation for behaviour of numerical and exact solutions with $m=6$. From this representation, we can see that the numerical solution is in a very good agreement with exact solution.

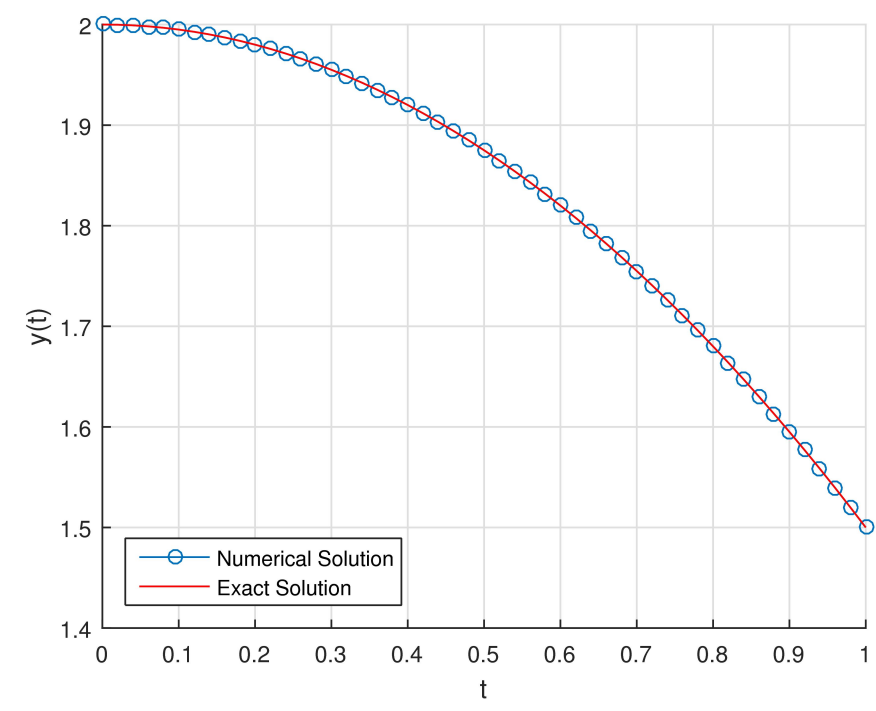

Figure 14. The behaviour of the numerical and exact solutions with $m=6$.

\subsection{Example 6}

For the last example, let us consider the below fractional differential equation [63]

$$
\begin{aligned}
y(t)+D^{1 / 2} y(t)-2 y(t) & =0, t \in(0, R] \\
y(0) & =1
\end{aligned}
$$

which arises, for example, in the study of generalized Basset force occuring when a spherical object sinks in a (relatively dense) incompressible viscous fluid; see [43,67]. By use of Laplace transformation of Caputo derivatives, we get the analytical solution as following

$$
y(t)=\frac{2}{3 \sqrt{t}} E_{1 / 2,1 / 2}(\sqrt{t})-\frac{1}{6 \sqrt{t}} E_{1 / 2,1 / 2}(-2 \sqrt{t})-\frac{1}{2 \sqrt{\pi t}},
$$

where the Mittag-Leffler function $E_{\lambda, \mu}(t)$ with parameters $\lambda, \mu>0$ is given as

$$
E_{\lambda, \mu}(t)=\sum_{k=0}^{\infty} \frac{t^{k}}{\Gamma(\lambda k+\mu)}
$$

This Mittag-Leffler function and its variations are very significant in fractional calculus and fractional differential equations [68].

In order to solve given problem by use of proposed method and compare the results, we take $t \in(0,5]$ and use different values of $\delta$ and $m$.

Table 9 lists the exact and obtained numerical solutions by use of presented method and method of [63] for the given problem for $m=5,10,15,20$. Comparison of this results shows that, even for small values of $m$, the numerical solution obtained by use of presented method is in a better agreement with exact solution. 
Table 9. The resulting values for Example 6, with $R=5$ in some values of $t$.

\begin{tabular}{cccccccccc}
\hline $\boldsymbol{t}$ & Exact & $\begin{array}{c}\text { Proposed } \\
\text { Method } \\
\boldsymbol{m = 5}\end{array}$ & $\begin{array}{c}\text { Method Given } \\
\text { in [63] } \\
\boldsymbol{m}=\mathbf{5}\end{array}$ & $\begin{array}{c}\text { Proposed } \\
\text { Method } \\
\boldsymbol{m}=\mathbf{1 0}\end{array}$ & $\begin{array}{c}\text { Method Given } \\
\text { in [63] } \\
\boldsymbol{m}=\mathbf{1 0}\end{array}$ & $\begin{array}{c}\text { Proposed } \\
\text { Method } \\
\boldsymbol{m}=\mathbf{1 5}\end{array}$ & $\begin{array}{c}\text { Method Given } \\
\text { in [63] } \\
\boldsymbol{m}=\mathbf{1 5}\end{array}$ & $\begin{array}{c}\text { Proposed } \\
\text { Method } \\
\boldsymbol{m}=\mathbf{2 0}\end{array}$ & $\begin{array}{c}\text { Method Given } \\
\text { in [63] } \\
\boldsymbol{m}=\mathbf{2 0}\end{array}$ \\
\hline 1 & 3.42445 & 3.42415 & 2.714336 & 3.425121 & 3.426525 & 3.42376044 & 3.42496 & 3.424563 \\
2 & 9.69088 & 9.670891 & 8.922571 & 9.692732 & 9.696794 & 9.68896761 & 9.692754 & 9.691185 \\
3 & 26.6414 & 26.60757 & 24.59981 & 26.64646 & 26.65929 & 26.6362145 & 26.64683 & 26.64225 \\
4 & 72.6729 & 72.53849 & 65.78029 & 72.68665 & 72.72038 & 72.6587861 & 72.68787 & 72.6752 \\
5 & 197.77 & 197.5757 & 180.1481 & 197.8077 & 197.8994 & 197.731934 & 197.8112 & 197.7766 \\
\hline
\end{tabular}

In Figures 15a, 16a and 17a, we present the graphical representation of comparison between exact solution and the numerical solutions obtained by using proposed method and the method of [63] with taking $m=5,10,20$ respectively. Also in Figures 15b, $16 \mathrm{~b}$ and $17 \mathrm{~b}$ we show the behaviour of absolute errors obtained by proposed method and the method of [63] in the interval [0,1] with $m=5,10,20$.
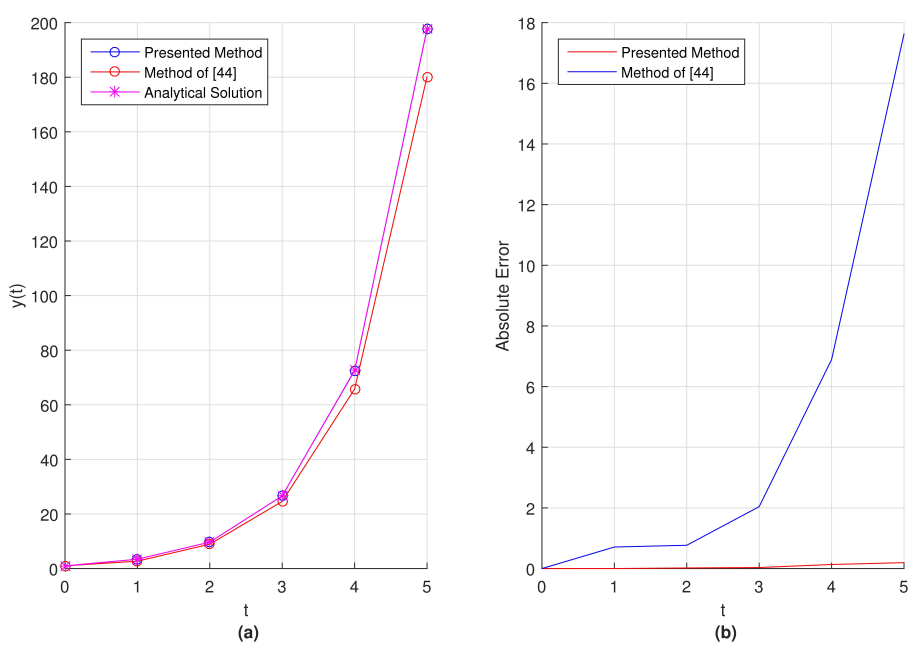

Figure 15. (a) The comparison of analytical solution and numerical solutions obtained by the proposed method and the method of [63] with $m=5$. (b) The behaviour of the absolute errors between the exact solution and numerical solutions obtained by our method and the method given in [63] with $m=5$.
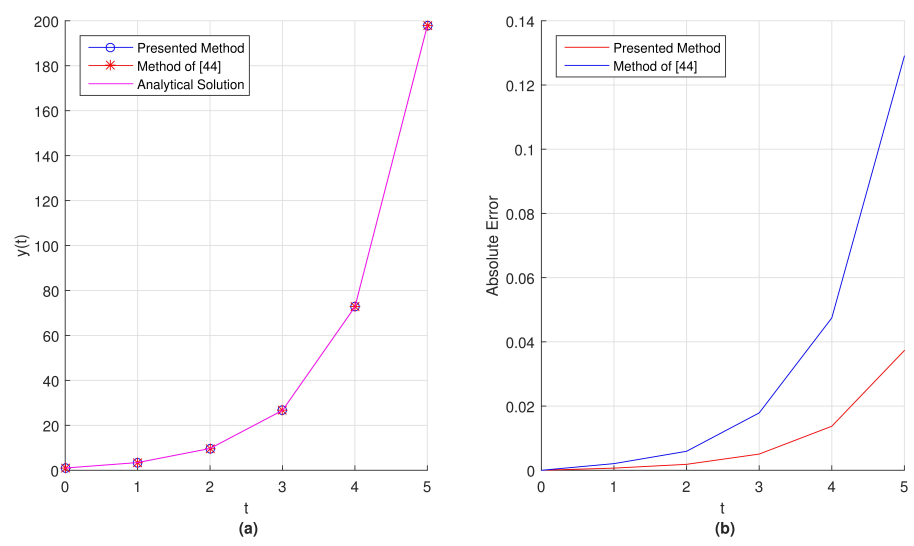

Figure 16. (a) The comparison of analytical solution and numerical solutions obtained by the proposed method and the method of [63] with $m=10$. (b) The behaviour of the absolute errors between the exact solution and numerical solutions obtained by our method and the method given in [63] with $m=10$. 

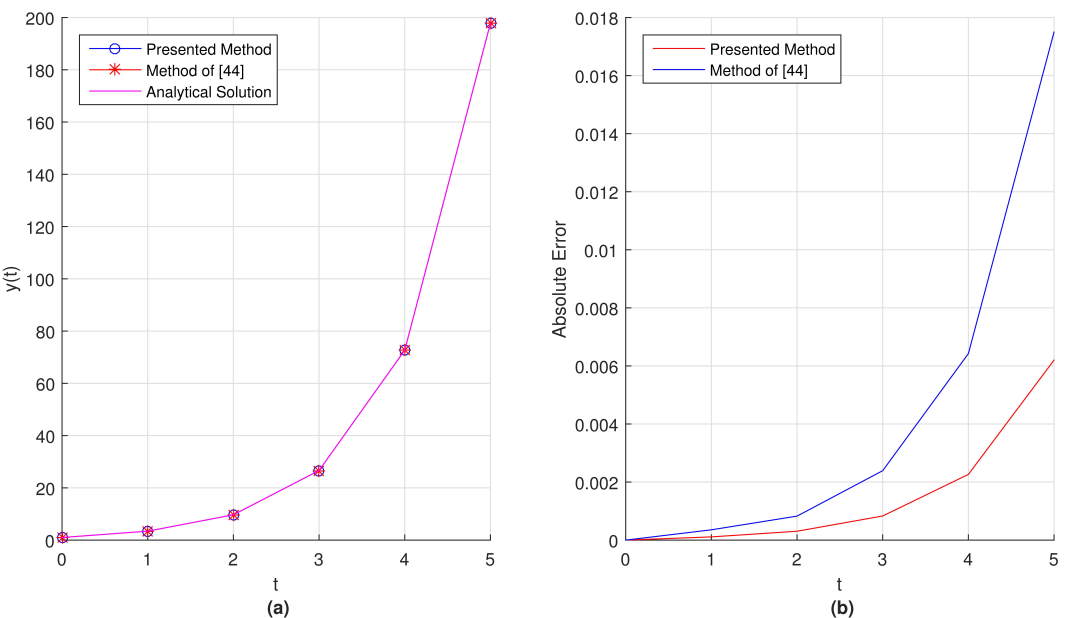

Figure 17. (a) The comparison of analytical solution and numerical solutions obtained by the proposed method and the method of [63] with $m=20$. (b) The behaviour of the absolute errors between the exact solution and numerical solutions obtained by our method and the method given in [63] with $m=20$.

From these graphical results represented in Figures 15-17, we can conclude that the absolute error obtained by our method is remaining smaller when compared the absolute error of method given in Reference [63].

\section{Conclusions}

In this work, an operational matrix based on the fractional Taylor vector is used to numerically solve the multi-term fractional differential equations by reducing them to a set of linear algebraic equations, which simplifies the problem. From comparison of the obtained results with exact solutions and also with results of other methods in the literature, we conclude that the proposed method provides the solution with high accuracy. The findings also show that, even for the small number of steps, we can get satisfactory results by using presented method. All computational results are obtained by using MATLAB.

Author Contributions: Formal analysis, İ.A.; Supervision, N.I.M. All authors contributed equally to this article. All authors have read and agreed to the published version of the manuscript.

Funding: This research received no external funding.

Conflicts of Interest: The authors declare no conflict of interest.

\section{References}

1. Oldham, K.B.; Spanier, J. The Fractional Calculus: Theory and Applications of Differentiation and Integration to Arbitrary Order; AP: New York, NY, USA, 1974.

2. Miller, K.S.; Ross, B. An Introduction to the Fractional Calculus and Fractional Differential Equations; Wiley: New York, NY, USA, 1993.

3. Samko, S.G.; Kilbas, A.A.; Marichev, O.I. Fractional Integrals and Derivatives: Theory and Applications; Gordan and Breach: New York, NY, USA, 1993.

4. Diethelm, K. The Analysis of Fractional Differential Equations; Springer: Heidelberg, Germany, 2010.

5. Machado, J.T.; Kiryakova, V.; Mainardi, F. Recent history of fractional calculus. Commun. Nonlinear Sci. Num. Simul. 2011, 16, 1140-1153. [CrossRef]

6. Hajipour, M.; Jajarmi, A.; Baleanu, D. An efficient nonstandard finite difference scheme for a class of fractional chaotic systems. J. Comput. Nonlinear Dyn. 2017, 13, 021013. [CrossRef]

7. Huang, L.; Bae, Y. Chaotic dynamics of the fractional-love model with an external environment. Entropy 2018, 20, 53. [CrossRef] 
8. Zheng, L.; Zhang, X. Modeling and Analysis of Modern Fluid Problems. Mathematics in Science and Engineering; AP: London, UK, 2017.

9. Mainardi, F. Fractional Calculus and Waves in Linear Viscoelasticity; ICP: London, UK, 2010.

10. Mahmudov, N.I. Finite-approximate controllability of evolution equations. Appl. Comput. Math. 2017, 16, 159-167.

11. Baleanu, D.; Jajarmi, A.; Hajipour, M. A new formulation of the fractional optimal control problems involving Mittag_Leffler nonsingular kernel. J. Optimiz. Theory. App. 2017, 175, 718-737. [CrossRef]

12. Singh, J.; Kumar, D.; Baleanu, D. On the analysis of chemical kinetics system pertaining to a fractional derivative with Mittag-Leffler type kernel. Chaos Interdiscip. J. Nonlinear Sci. 2017, 27, 103113. [CrossRef]

13. Stoenoiu, C.E.; Bolboacă, S.D.; Jäntschi, L. Model formulation and interpretation-from experiment to theory. Int. J. Pure Appl. Math. 2008, 47, 9-16.

14. Oldham, K.B. Fractional differential equations in electrochemistry. Adv. Eng. Softw. 2010, 41, 9-12. [CrossRef]

15. Ertürk, V.S.; Odibat, Z.M.; Momani, S. An approximate solution of a fractional order differential equation model of human T-cell lymphotropic virus I (HTLV-I) infection of CD4+ T-cells. Comput. Math. Appl. 2011, 62, 996-1002. [CrossRef]

16. Hilfer, R. Applications of Fractional Calculus in Physics; World Scientific Press: Singapore, 2000.

17. Magin, R.L. Fractional Calculus in Bioengineering; Begell House Publishers: Redding, CA, USA, 2006.

18. Fallahgoul, H.; Focardi, S.; Fabozzi, F. Fractional Calculus and Fractional Processes With Applications to Financial Economics: Theory and Application; AP: Cambridge, MA, USA, 2016.

19. Baleanu, D.; Lopes, A.M. (Eds.) Handbook of Fractional Calculus with Applications, Volume 8: Applications in Engineering, Life and Social Sciences, Part B; De Gruyter: Berlin, Germany, 2019.

20. Tarasov, V.E. On history of mathematical economics: Application of fractional calculus. Mathematics 2019, 7, 509. [CrossRef]

21. Ming, H.; Wang, J.; Fečkan, M. The Application of Fractional Calculus in Chinese Economic Growth Models. Mathematics 2019, 7, 665. [CrossRef]

22. Esen, A.; Sulaiman, T.A.; Bulut, H.; Baskonus, H.M. Optical solitons and other solutions to the conformable space-time fractional Fokas-Lenells equation. Optik 2018, 167, 150-156 [CrossRef]

23. Zabadal, J.; Vilhena, M.; Livotto, P. Simulation of chemical reactions using fractional derivatives. Nuovo Cimento. B 2001, 116, 529-545.

24. Yang, F.; Zhu, K.Q. On the definition of fractional derivatives in rheology. Theor. Appl. Mech. Lett. 2011, 1, 012007. [CrossRef]

25. Diethelm, K.; Ford, N.J.; Freed, A.D. A predictor-corrector approach for the numerical solution of fractional differential equations. Nonlinear Dyn. 2002, 29, 3-22. [CrossRef]

26. Podlubny, I. Fractional Differential Equations; AP: New York, NY, USA, 1999.

27. Çenesiz, Y.; Keskin, Y.; Kurnaz, A. The solution of the Bagley-Torvik equation with the generalized Taylor collocation method. J. Franklin Inst. 2010, 347, 452-466. [CrossRef]

28. Ray, S.S.; Bera, R.K. Solution of an extraordinary differential equation by Adomian decomposition method. J. Appl. Math. 2004, 2004, 331-338. [CrossRef]

29. Vanani, S.K.; Aminataei, A. Tau approximate solution of fractional partial differential equations. Comput. Math. Appl. 2011, 62, 1075-1083. [CrossRef]

30. Rani, D.; Mishra, V.; Cattani, C. Numerical inverse Laplace transform for solving a class of fractional differential equations. Symmetry 2019, 11, 530. [CrossRef]

31. Khashan, M.M.; Amin, R.; Syam, M.I. A new algorithm for fractional Riccati type differential equations by using Haar wavelet. Mathematics 2019, 7, 545. [CrossRef]

32. Li, Y.; Sun, N. Numerical solution of fractional differential equations using the generalized block pulse operational matrix. Comput. Math. Appl. 2011, 62, 1046-1054. [CrossRef]

33. Saadatmandi, A.; Dehghan, M. A tau approach for solution of the space fractional diffusion equation. Comput. Math. Appl. 2011, 62, 1135-1142. [CrossRef]

34. Abuasad, S.; Yildirim, A.; Hashim, I.; Karim, A.; Ariffin, S.; Gómez-Aguilar, J.F. Fractional multi-step differential transformed method for approximating a fractional stochastic sis epidemic model with imperfect vaccination. Int. J. Environ. Res. Public Health 2019, 16, 973. [CrossRef] [PubMed]

35. Veeresha, P.; Prakasha, D.G.; Baleanu, D. An efficient numerical technique for the nonlinear fractional Kolmogorov-Petrovskii-Piskunov equation. Mathematics 2019, 7, 265. [CrossRef] 
36. Silva, F.; Moreira, D.; Moret, M. Conformable Laplace transform of fractional differential equations. Axioms 2018, 7, 55. [CrossRef]

37. Pitolli, F. A fractional B-spline collocation method for the numerical solution of fractional predator-prey models. Fractal Fract. 2018, 2, 13. [CrossRef]

38. Fazio, R.; Jannelli, A.; Agreste, S. A Finite Difference Method on Non-Uniform Meshes for Time-Fractional Advection-Diffusion Equations with a Source Term. Appl. Sci. 2018, 8, 960. [CrossRef]

39. Odibat, Z.; Momani, S.; Xu, H. A reliable algorithm of homotopy analysis method for solving nonlinear fractional differential equations. Appl. Math. Model. 2010, 34, 593-600. [CrossRef]

40. Krishnasamy, V.S.; Razzaghi, M. The numerical solution of the Bagley-Torvik equation with fractional Taylor method. J. Comput. Nonlin. Dyn. 2016, 11. [CrossRef]

41. Krishnasamy, V.S.; Mashayekhi, S.; Razzaghi, M. Numerical solutions of fractional differential equations by using fractional Taylor basis. IEEE/CAA J. Autom. Sin. 2017, 4, 98-106.

42. Jiang, H.; Liu, F.; Turner, I.; Burrage, K. Analytical solutions for the multi-term time-fractional diffusion-wave/diffusion equations in a finite domain. Comput. Math. Appl. 2012, 64, 3377-3388. [CrossRef]

43. Basset, A.B. On the descent of a sphere in a vicous liquid. Quart. J. 1910, 41, 369-381.

44. Torvik, P.J.; Bagley, R.L. On the Appearance of the fractional derivative in the behavior of real materials. ASME J. Appl. Mech. 1984, 51, 294-298. [CrossRef]

45. Mahmudov, N.I.; Emin, S.; Bawanah, S. On the Parametrization of Caputo-Type Fractional Differential Equations with Two-Point Nonlinear Boundary Conditions. Mathematics 2019, 7, 707. [CrossRef]

46. Diethelm, K.; Ford, N.J. Analysis of fractional differential equations. J. Math. Anal. Appl. 2002, 265, $229-248$. [CrossRef]

47. Daftardar-Gejji, V.; Jafari, H. Analysis of a system of nonautonomous fractional differential equations involving Caputo derivatives. J. Math. Anal. Appl. 2007, 328, 1026-1033. [CrossRef]

48. Aphithana, A.; Ntouyas, S.K.; Tariboon, J. Existence and uniqueness of symmetric solutions for fractional differential equations with multi-order fractional integral conditions. Bound. Value Probl. 2015, 2015, 68. [CrossRef]

49. Aliev, F.A.; Aliev, N.A.; Safarova, N.A. Transformation of the Mittag-Leffler Function to an Exponential Function and Some of its Applications to Problems with a Fractional Derivative. Appl. Comput. Math 2019, 18, 316-325.

50. Diethelm, K.; Ford, N.J.; Freed, A.D. Detailed error analysis for a fractional Adams method. Numer. Algorithms 2004, 36, 31-52. [CrossRef]

51. Lepik, Ü. Solving fractional integral equations by the Haar wavelet method. Appl. Math. Comput. 2009, 214, 468-478. [CrossRef]

52. Arikoglu, A.; Ozkol, I. Solution of fractional differential equations by using differential transform method. Chaos Soliton. Fract. 2007, 34, 1473-1481. [CrossRef]

53. Diethelm, K.; Ford, N.J. Multi-order fractional differential equations and their numerical solution. Appl. Math. Comput. 2004, 154, 621-640. [CrossRef]

54. Saw, V.; Kumar, S. The approximate solution for multi-term the fractional order initial value problem using collocation method based on shifted Chebyshev polynomials of the first kind. In Information Technology and Applied Mathematics; Chandra, P., Giri, D., Li, F., Kar, S., Jana, D.K., Eds.; Springer: Singapore, 2019; Volume 699, pp. 53-67.

55. Bolandtalat, A.; Babolian, E.; Jafari, H. Numerical solutions of multi-order fractional differential equations by Boubaker polynomials. Open Phys. 2016, 14, 226-230. [CrossRef]

56. Popolizio, M. Numerical solution of multiterm fractional differential equations using the matrix Mittag-Leffler functions. Mathematics 2018, 6, 7. [CrossRef]

57. Rebenda, J. Application of Differential Transform to Multi-Term Fractional Differential Equations with Non-Commensurate Orders. Symmetry 2019, 11, 1390. [CrossRef]

58. Garrappa, R. Numerical solution of fractional differential equations: A survey and a software tutorial. Mathematics 2018, 6, 16. [CrossRef]

59. Han, W.; Chen, Y.M.; Liu, D.Y.; Li, X.L.; Boutat, D. Numerical solution for a class of multi-order fractional differential equations with error correction and convergence analysis. Adv. Differ. Equ. N. Y. 2018, 2018, 253. [CrossRef] 
60. El-Mesiry, A.E.M.; El-Sayed, A.M.A.; El-Saka, H.A.A. Numerical methods for multi-term fractional (arbitrary) orders differential equations. Appl. Math. Comput. 2005, 160, 683-699. [CrossRef]

61. Bhrawy, A.H.; Tharwat, M.M.; Yildirim, A. A new formula for fractional integrals of Chebyshev polynomials: Application for solving multi-term fractional differential equations. Appl. Math. Model. 2013, 37, 4245-4252. [CrossRef]

62. Deng, W.H.; Li, C. Numerical schemes for fractional ordinary differential equations. Numer. Model. 2012, 16, 355-374.

63. Esmaeili, S.; Shamsi, M. A pseudo-spectral scheme for the approximate solution of a family of fractional differential equations. Commun. Nonlinear Sci. 2011, 16, 3646-3654. [CrossRef]

64. Deng, W. Short memory principle and a predictor-corrector approach for fractional differential equations. J. Comput. Appl. Math. 2007, 206, 174-188. [CrossRef]

65. Maleknejad, K.; Nouri, K.; Torkzadeh, L. Operational matrix of fractional integration based on the shifted second kind Chebyshev polynomials for solving fractional differential equations. Med. J. Math. 2016, 13, 1377-1390. [CrossRef]

66. Liu, J.; Li, X.; Wu, L. An operational matrix of fractional differentiation of the second kind of Chebyshev polynomial for solving multiterm variable order fractional differential equation. Math. Probl. Eng. 2016, 2016, 7126080. [CrossRef]

67. Carpinteri, A.; Mainardi, F. (Eds.) Fractional calculus: Some basic problems in continuum and statistical mechanics. In Fractals and Fractional Calculus in Continuum Mechanics; Springer Verlag: Wien, Austria; New York, NY, USA, 1997; pp. 291-348.

68. Srivastava, H.M.; Fernandez, A.; Baleanu, D. Some New Fractional-Calculus Connections between Mittag-Leffler Functions. Mathematics 2019, 7, 485. [CrossRef]

(C) 2020 by the authors. Licensee MDPI, Basel, Switzerland. This article is an open access article distributed under the terms and conditions of the Creative Commons Attribution (CC BY) license (http:/ / creativecommons.org/licenses/by/4.0/). 